\title{
Exact solutions of forced Burgers equations with time variable coefficients
}

\author{
Şirin A. Büyükaşı* , Oktay K. Pashaev \\ Dept. of Mathematics, Izmir Institute of Technology, 35430 Urla, Izmir, Turkey
}

\section{A R T I C L E I N F O}

\section{Article history:}

Received 25 April 2012

Received in revised form 25 July 2012

Accepted 30 November 2012

Available online 12 December 2012

\section{Keywords:}

Forced Burgers equation

Variable parameters

C-integrable

Shock waves

Pole dynamics

Exact solutions

\begin{abstract}
A B S T R A C T
In this paper, we consider a forced Burgers equation with time variable coefficients of the form $U_{t}+(\dot{\mu}(t) / \mu(t)) U+U U_{x}=(1 / 2 \mu(t)) U_{x x}-\omega^{2}(t) x$, and obtain an explicit solution of the general initial value problem in terms of a corresponding second order linear ordinary differential equation. Special exact solutions such as generalized shock and multi-shock waves, triangular wave, $N$-wave and rational type solutions are found and discussed. Then, we introduce forced Burgers equations with constant damping and an exponentially decaying diffusion coefficient as exactly solvable models. Different type of exact solutions are obtained for the critical, over and under damping cases, and their behavior is illustrated explicitly. In particular, the existence of inelastic type of collisions is observed by constructing multi-shock wave solutions, and for the rational type solutions the motion of the pole singularities is described.
\end{abstract}

(c) 2012 Elsevier B.V. All rights reserved.

\section{Introduction}

The nonlinear diffusion equation, known as Burgers equation (BE) after the extensive work of Burgers [1,2] is an important model which appears in various fields of physical science. In hydrodynamics, it is a standard model of turbulence used to study propagation of nonlinear waves and shock formation [3]. It is used also to describe processes in gas dynamics [4,5], nonlinear acoustics [6], heat conduction, and plasma physics. In cosmology, the Burgers equation is a good approximation to understand the formation and distribution of matter at large scales [7].

The standard Burgers equation is of the form $V_{t}+V V_{x}=v V_{x x}$, where $V$ mostly represents the velocity field, $t$ is a time variable, $x \in \mathbb{R}$ is the space variable and $v$ is a constant viscosity or diffusion coefficient. This equation is probably the simplest nonlinear model admitting direct linearization, and thus being C-integrable in contrast to S-integrable systems which require spectral transform technics. Indeed, analytic solutions of the Burgers equation can be obtained by the Cole-Hopf transformation, which transforms the nonlinear Burgers equation to a linear heat equation [4,5]. Lately, other methods like, Hirota's direct method and Bäcklund transformation [11], hyperbolic function method [8], and homogeneous balance method [9] were used to construct new exact solutions of the BE.

As known, the main features of the Burgers equation are due to the simultaneous existence of a nonlinear term and a linear diffusion term. If the diffusion is dominant over nonlinearity, the solution of the BE approaches the solution of the diffusion equation. On the other hand, if the nonlinear term dominates over the diffusion, one may expect formation of shock discontinuities. An interesting property of the BE appears when a balance occurs between the nonlinear effect and the effects of dissipative nature. In that case, the system exhibits shock profile solitary wave solutions. Moreover, Burgers equation posses also multi-shock solitary wave solutions [10,11] and shocks of different amplitude and speed can fuse (merge) to a single shock, so that completely non-elastic interactions may occur. Another important property of the Burgers equation

\footnotetext{
* Corresponding author.

E-mail addresses: sirinatilgan@iyte.edu.tr (Ş.A. Büyükaşı), oktaypashaev@iyte.edu.tr (O.K. Pashaev).
} 
is related with the rational type solutions. Indeed, the zeros of the heat equation solution lead to pole singularities for the Burgers solution. Choodnovsky brothers [12] and Calogero [13], showed that the motion of these poles corresponds formally to the motion of one-dimensional particles interacting via simple two-body potentials, such that the corresponding many body problems are integrable. For recent work on the pole dynamics of the standard Burgers equation one can see [14].

The standard BE as mentioned above is exactly solvable model, however the inhomogeneous and variable parametric Burgers equations, in general, are not integrable and very few exactly solvable models are known. For example, in case $U_{t}+U U_{x}=v U_{x x}+F(x, t)$, if the forcing term depends only on time, i.e., $F(x, t)=G(t)$, this equation can be transformed to a standard Burgers equation, see [15]. The problem with an elastic forcing term $F(x, t)=-k^{2} x+f(t)$ is discussed and analytic solutions are obtained in [16]. Later, the problem with $F(x, t)=G(t) x$, where $G(t)$ is arbitrary, was solved in [17]. In [18], an invertible transformation between the inhomogeneous BE and the stationary Schrödinger equation was constructed so that each solution of the stationary Schrödinger equation generated a fully time-dependent solution of the inhomogeneous BE. Recently, exact solutions were obtained using the Cole-Hopf transformation and the Green's function approach, see [19]. Transformation properties of a variable-coefficient Burgers equation were discussed in [20]. In [21], a forced Burgers model with space- and time-dependent coefficients of the form $U_{t}+a(x, t) U U_{x}=b(x, t) U_{x x}+F(x, t)$, was investigated using a generalized Cole-Hopf transform and symbolic computation. For the significance of the generalized forced Burgers models and possible applications in various fields one can see again the discussion in [21] and references given there.

In this work, we consider a forced Burgers equation (FBE) with time variable coefficients of the form

$$
U_{t}+\frac{\dot{\mu}(t)}{\mu(t)} U+U U_{x}=\frac{1}{2 \mu(t)} U_{x x}-\omega^{2}(t) x, \quad-\infty<x<\infty
$$

where $\Gamma(t)=\dot{\mu}(t) / \mu(t)$ is the damping term, $D(t)=1 / 2 \mu(t)$ is the diffusion coefficient, and $F(x, t)=-\omega^{2}(t) x$ is the forcing term which is linear in the space variable $x$. In fact, this equation for constant $\mu(t)$, or under change of variables $t^{\prime}=\int^{t} d s / \mu(s), v\left(x, t^{\prime}\right)=\mu(t) U(x, t)$ reduces to forced Burgers equation of type $v_{t^{\prime}}+v v_{x}=(1 / 2) v_{x x}+G\left(t^{\prime}\right) x$, which was studied by Eule and Friedrich in [17]. However, although in [17] solution was given for an arbitrary function $G\left(t^{\prime}\right)$, only the cases when $G\left(t^{\prime}\right)$ is a constant and the case when it is a stochastic white noise force were discussed.

Motivated by this idea, here we introduce and give explicit exact solutions for forced Burgers equations with different variable parameters. For this, in Section 2 first we establish the relation between the FBE (1) and the standard BE. As a result, explicit solution for the IVP of the FBE (1) is found in terms of solutions to the heat equation and a second order linear ODE. Then, some particular exact solutions such as shock and multi-shock type waves, triangular wave, $N$-wave and rational type solutions are given. In Section 3, for comparative reasons, first we recall some solutions of the forced Burgers equation with constant $\mu$ and $\omega^{2}$. Then, exactly solvable models (1) with positive constant damping and exponentially decaying diffusion coefficient are considered and different type of exact solutions mentioned in Section 2 are obtained for the critical, under and over damping cases. We observe generalized traveling wave solutions which speed, steepness, and shock amplitude are functions of time. Special properties like interaction of shocks in multi-shock wave solutions, and motion of pole singularities of rational type solutions are described explicitly and illustrated constructively. Section 4 includes brief summary, future plans and potential fields of applications.

\section{Forced Burgers equation with time dependent coefficients}

In this section, we obtain relation between solution of the FBE with variable coefficients and the standard Burgers equation. Then, using Cole-Hopf transform we find an analytic solution of the IVP for the FBE (1) in terms of solution to a corresponding second order linear ODE.

Proposition 2.1. If $r(t)$ is solution of the IVP for the linear ODE

$$
\ddot{r}+\frac{\dot{\mu}(t)}{\mu(t)} \dot{r}+\omega^{2}(t) r=0, \quad r\left(t_{0}\right)=r_{0} \neq 0, \quad \dot{r}\left(t_{0}\right)=0,
$$

then the IVP for the FBE with variable coefficients

$$
\left\{\begin{array}{l}
U_{t}+\frac{\dot{\mu}(t)}{\mu(t)} U+U U_{x}=\frac{1}{2 \mu(t)} U_{x x}-\omega^{2}(t) x \\
\left.U(x, t)\right|_{t=t_{0}}=U\left(x, t_{0}\right), \quad-\infty<x<\infty
\end{array}\right.
$$

has solution in the following forms:

$$
\text { (a) } U(x, t)=\frac{\dot{r}(t)}{r(t)} x+\frac{r\left(t_{0}\right)}{\mu(t) r(t)} V(\eta(x, t), \tau(t)) \text {, }
$$

where

$$
\eta(x, t)=\frac{r\left(t_{0}\right)}{r(t)} x ; \quad \tau(t)=r^{2}\left(t_{0}\right) \int^{t} \frac{d \xi}{\mu(\xi) r^{2}(\xi)}, \quad \tau\left(t_{0}\right)=0
$$


and the function $V(\eta, \tau)$ satisfies the IVP for the standard $B E$

$$
\begin{aligned}
& \left\{\begin{array}{l}
V_{\tau}+V V_{\eta}=\frac{1}{2} V_{\eta \eta}, \\
V(\eta, 0)=\mu\left(t_{0}\right) U\left(\eta, t_{0}\right) .
\end{array}\right. \\
& \text { (b) } U(x, t)=\frac{\dot{r}(t)}{r(t)} x-\frac{r\left(t_{0}\right)}{\mu(t) r(t)} \frac{\varphi_{\eta}(\eta(x, t), \tau(t))}{\varphi(\eta(x, t), \tau(t))},
\end{aligned}
$$

where $\eta, \tau$ are as defined in part $(a)$, and $\varphi(\eta, \tau)$ satisfies the IVP for the heat equation

$$
\left\{\begin{array}{l}
\varphi_{\tau}=\frac{1}{2} \varphi_{\eta \eta}, \\
\varphi(\eta, 0)=\exp \left[-\int^{\eta} \mu\left(t_{0}\right) U\left(\xi, t_{0}\right) d \xi\right] .
\end{array}\right.
$$

Proof. (a) Using substitution $U(x, t)=[\mu(t)]^{-1}[\rho(t) x+s(t) V(s(t) x, \tau(t))]$, one can show that, if the auxiliary functions satisfy the nonlinear system of ordinary differential equations

$$
\begin{aligned}
& \dot{\rho}+\frac{\rho^{2}}{\mu(t)}+\mu(t) \omega^{2}(t)=0, \quad \rho\left(t_{0}\right)=0, \\
& \dot{\tau}-\frac{s^{2}}{\mu(t)}=0, \quad \tau\left(t_{0}\right)=0, \\
& \dot{s}+\frac{\rho(t)}{\mu(t)} s=0, \quad s\left(t_{0}\right)=1,
\end{aligned}
$$

then the IVP (3) for the FBE transforms to the IVP (6) for the standard BE. Also, noticing that Eq. (9) is a nonlinear Riccati equation, the system is easily solved, and we obtain the functions

$$
\rho(t)=\mu(t) \frac{\dot{r}(t)}{r(t)} ; \quad \tau(t)=r^{2}\left(t_{0}\right) \int^{t} \frac{d \xi}{\mu(\xi) r^{2}(\xi)}, \quad \tau\left(t_{0}\right)=0 ; \quad s(t)=\frac{r\left(t_{0}\right)}{r(t)},
$$

which substituted back give the result (4). Thus, solution of the FBE (3) is explicitly obtained in terms of solution $V(\eta, \tau)$ to the BE (6) and solution $r(t)$ of the IVP for the linear ODE (2). Part (b) of the proposition, follows directly from the Cole-Hopf transformation $V=-\varphi_{\eta} / \varphi$, which reduces the IVP (6) for the BE to the IVP (8) for the usual heat equation.

The solution of the IVP (8) for the heat equation

$$
\varphi(\eta, \tau)=\frac{1}{\sqrt{2 \pi \tau}} \int_{-\infty}^{\infty} \exp \left[-\frac{(\eta-\xi)^{2}}{2 \tau}\right] \varphi(\xi, 0) d \xi,
$$

and Cole-Hopf transformation $V=-\varphi_{\eta} / \varphi$, leads to solution of the IVP (6) for the BE

$$
V(\eta, \tau)=\frac{\int_{-\infty}^{\infty}\left(\frac{\eta-\xi}{\tau}\right) \exp \left[-\left(\frac{(\eta-\xi)^{2}}{2 \tau}+\int^{\xi} V\left(\xi^{\prime}, 0\right) d \xi^{\prime}\right)\right] d \xi}{\int_{-\infty}^{\infty} \exp \left[-\left(\frac{(\eta-\xi)^{2}}{2 \tau}+\int^{\xi} V\left(\xi^{\prime}, 0\right) d \xi^{\prime}\right)\right] d \xi} .
$$

Therefore, using the above proposition, one can find formal solution of the IVP (3) for the FBE in terms of solution $r(t)$ of the linear ODE (2), that is

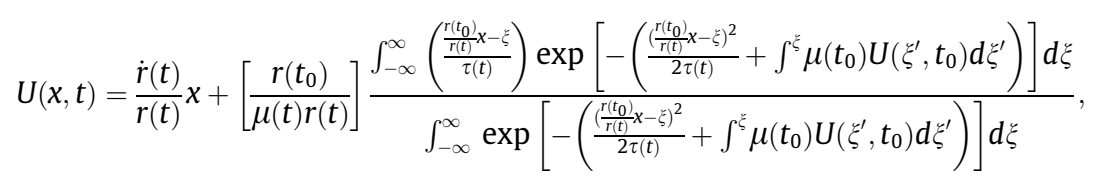

where $\tau(t)$ is as defined in (5), and the time interval on which the solution exists depends on the properties of the auxiliary functions. Since it is difficult to analyze solution (11) for an arbitrary initial condition, in what follows, we consider particular problems for which the FBE (3) subject to some localized initial profiles has exact solutions and one can observe explicitly their behavior. As known, the standard BE (6) has different type of solutions, such as shock solitary waves, similarity, $N$-wave and rational function solutions. This suggests us to look for corresponding type of solutions for the FBE with variable coefficients, as follows.

(a) Shock wave solutions. The standard BE (6) has shock solitary wave solution

$$
V(\eta, \tau)=c-A \tanh \left[A\left(\eta-c \tau+c_{0}\right)\right]
$$


where $A, c, c_{0}$ are arbitrary real constants. Then, the FBE (3) with initial condition

$$
U\left(x, t_{0}\right)=\frac{1}{\mu\left(t_{0}\right)}\left(c-A \tanh \left[A x+c_{0}\right]\right)
$$

has generalized shock wave solution

$$
U(x, t)=\frac{\dot{r}(t)}{r(t)} x+\frac{r\left(t_{0}\right)}{\mu(t) r(t)}\left(c-A \tanh \left[A\left(\frac{r\left(t_{0}\right)}{r(t)} x-c \tau(t)+c_{0}\right)\right]\right),
$$

where $r(t)$ is a solution of the IVP (2) and $\tau(t)$ is given in (5). In particular, when in (13) one has $c=c_{0}=0$, the FBE (3) has shock type static solution of the form

$$
U(x, t)=\frac{\dot{r}(t)}{r(t)} x-\frac{A r\left(t_{0}\right)}{\mu(t) r(t)} \tanh \left[\frac{\operatorname{Ar}\left(t_{0}\right)}{r(t)} x\right],
$$

corresponding to the initial condition $U\left(x, t_{0}\right)=-\left(A / \mu\left(t_{0}\right)\right) \tanh [A x],-\infty<x<\infty$.

Solution (12) of the standard BE (6) is a localized wave of constant amplitude moving with constant speed. However, the solution (13) of the FBE with variable coefficients is a generalized traveling wave of the form $U(x, t)=\tilde{u}(x, t)+$ $A(t) \tanh [B(t)(x-C(t))]$, where the term $\tilde{u}(x, t)$ contributes to the wave amplitude, $A(t)$ is the shock amplitude, $B(t)$ is related with the steepness of the shock profile, $x=C(t)$ describes the motion of the "center" of the profile, and $v=\dot{C}(t)$ is its velocity. Accordingly, for the wave solution (13), one can see that the shock amplitude is proportional to $1 / \mu(t) r(t)$, and steepness of the profile is proportional to $1 / r(t)$. Also, the position of the "center" of the wave profile is described by $x(t)=\left[r(t) / r\left(t_{0}\right)\right]\left(c \tau(t)-c_{0}\right)$, where its velocity can be easily found using that $v(t)=\dot{x}(t)$.

Multi-shock wave solutions. Since the standard BE has multi-shock solitary wave solutions [10,11], it is natural to ask for multi-shock type solutions for the FBE with variable coefficients. Here, we outline the procedure, and give formal results. Clearly, the heat Eq. (8), has simple solutions of the form

$$
\varphi_{i}(\eta, \tau)=\exp \left[p_{i}(\eta, \tau)\right], \quad p_{i}(\eta, \tau)=-a_{i} \eta+\frac{a_{i}^{2}}{2} \tau+p_{i}^{0}, \quad a_{i}, p_{i}^{0} \in \mathbb{R},
$$

and their linear superposition

$$
\varphi(\eta, \tau)=\exp \left[p_{1}(\eta, \tau)\right]+\exp \left[p_{2}(\eta, \tau)\right]+\cdots+\exp \left[p_{k}(\eta, \tau)\right], \quad i=1,2, \ldots, k,
$$

is also a solution. By the Cole-Hopf transform $V=-\varphi_{\eta} / \varphi$, it follows that the BE (6), has corresponding solutions of the form

$$
V(\eta, \tau)=\frac{a_{1} \exp \left[p_{1}(\eta, \tau)\right]+a_{2} \exp \left[p_{2}(\eta, \tau)\right]+\cdots+a_{k} \exp \left[p_{k}(\eta, \tau)\right]}{\exp \left[p_{1}(\eta, \tau)\right]+\exp \left[p_{2}(\eta, \tau)\right]+\cdots+\exp \left[p_{k}(\eta, \tau)\right]}
$$

Therefore, using Proposition 2.1, one obtains that the FBE (3), has generalized multi-shock wave solutions given by

$$
U(x, t)=\frac{\dot{r}(t)}{r(t)} x+\frac{r\left(t_{0}\right)}{\mu(t) r(t)}\left[\frac{a_{1} \exp \left[p_{1}(x, t)\right]+a_{2} \exp \left[p_{2}(x, t)\right]+\cdots+a_{k} \exp \left[p_{k}(x, t)\right]}{\exp \left[p_{1}(x, t)\right]+\exp \left[p_{2}(x, t)\right]+\cdots+\exp \left[p_{k}(x, t)\right]}\right],
$$

where

$$
p_{i}(x, t)=-a_{i} \frac{r\left(t_{0}\right)}{r(t)} x+\frac{a_{i}^{2}}{2} \tau(t)+p_{i}^{0}, \quad i=1,2, \ldots, k,
$$

$r(t)$ is solution of (2), and $\tau(t)$ is given in (5). For $k=2$ and proper choice of constants, one obtains one-shock wave. When $k>2$ one expects formation of multi-shock wave solutions. Indeed, this is the case, and illustrative examples are given in Section 3 .

(b) Triangular wave solution. The BE (6) has triangular wave (similarity) solution,

$$
V(\eta, \tau)=\frac{1}{\sqrt{2 \pi \tau}}\left(\frac{\left(e^{A}-1\right) \exp \left[-\eta^{2} / 2 \tau\right]}{1+\frac{1}{2}\left(e^{A}-1\right) \operatorname{erfc}[\eta / \sqrt{2 \tau}]}\right),
$$

corresponding to initial condition $V(\eta, 0)=A \delta(\eta)$, see [10], where $A$ is a constant, $\delta(\eta)$ is the Dirac-delta distribution, and $\operatorname{erfc}[a]=(2 / \sqrt{\pi}) \int_{a}^{\infty} \exp \left[-\xi^{2}\right] d \xi$. Then, the FBE (3) has generalized triangular wave solution of the form

$$
U(x, t)=\frac{\dot{r}(t)}{r(t)} x+\frac{r\left(t_{0}\right)}{\mu(t) r(t)} \frac{1}{\sqrt{2 \pi \tau(t)}}\left(\frac{\left(e^{A}-1\right) \exp \left[-\left(\frac{r\left(t_{0}\right)}{r(t)} x\right)^{2} / 2 \tau(t)\right]}{1+\frac{1}{2}\left(e^{A}-1\right) \operatorname{erfc}\left[\frac{r\left(t_{0}\right)}{r(t)} x / \sqrt{2 \tau(t)}\right]}\right), \quad \tau(t)>0 .
$$

(c) $N$-wave solution. The heat Eq. (8) has solution

$$
\varphi(\eta, \tau)=1+\sqrt{\frac{a}{\tau}} \exp \left[-\eta^{2} / 2 \tau\right], \quad \tau>0
$$


which behaves like delta distribution as $\tau \rightarrow 0$ ( $a$-positive constant). The corresponding $N$-wave solution of BE (6), see [10], is

$$
V(\eta, \tau)=\left(\frac{\eta}{\tau}\right) \frac{\sqrt{a / \tau} \exp \left[-\eta^{2} / 2 \tau\right]}{1+\sqrt{a / \tau} \exp \left[-\eta^{2} / 2 \tau\right]}, \quad \tau>0
$$

Therefore, generalized $\mathrm{N}$-wave solution of the FBE (3) is of the form

$$
U(x, t)=\frac{\dot{r}(t)}{r(t)} x+\frac{r^{2}\left(t_{0}\right) x}{\mu(t) \tau(t) r^{2}(t)}\left(\frac{\sqrt{a / \tau(t)} \exp \left[-\left(\frac{r\left(t_{0}\right)}{r(t)} x\right)^{2} / 2 \tau(t)\right]}{1+\sqrt{a / \tau(t)} \exp \left[-\left(\frac{r\left(t_{0}\right)}{r(t)} x\right)^{2} / 2 \tau(t)\right]}\right), \quad \tau(t)>0 .
$$

Since the behavior of this solution at $\tau\left(t_{0}\right)=0$ is rather complicated, as an initial profile one can consider a profile at any time $t>t_{0}$.

(d) Rational function solutions. Formal solution of the IVP for the heat Eq. (8) can be found also by applying the evolution operator to the initial condition, that is

$$
\varphi(\eta, \tau)=\exp \left[(\tau / 2) \partial_{\eta}^{2}\right] \varphi(\eta, 0)=\sum_{k=0}^{\infty} \frac{1}{k !}\left(\frac{\tau}{2}\right)^{k} \partial_{\eta}^{2 k} \varphi(\eta, 0)
$$

If the initial condition is $\varphi_{m}(\eta, 0)=\eta^{m}$, then the solution of the heat problem (8) is

$$
\varphi_{m}(\eta, \tau)=\exp \left[(\tau / 2) \partial_{\eta}^{2}\right] \eta^{m}=H_{m}(\eta, \tau / 2), \quad m=0,1,2, \ldots,
$$

where $H_{m}(\eta, \tau / 2)$ are Kampè de Feriet polynomials, defined by

$$
H_{m}(\eta, \tau / 2)=m ! \sum_{k=0}^{[m / 2]} \frac{(\tau / 2)^{k}}{k !(m-2 k) !} \eta^{m-2 k}, \quad H_{m}(\eta, 0)=\eta^{m},
$$

with $[m / 2]=m / 2$ for even $m$, and $[m / 2]=(m-1) / 2$ for odd $m$, see [24]. Using also the relation $\partial_{\eta} H_{m}(\eta, \tau / 2)=m H_{m-1}(\eta, \tau / 2)$, it follows that, the $\mathrm{BE}(6)$ has rational solutions of the form

$$
V_{m}(\eta, \tau)=-\frac{\partial}{\partial \eta}\left[\ln \varphi_{m}(\eta, \tau)\right]=-\frac{m H_{m-1}(\eta, \tau / 2)}{H_{m}(\eta, \tau / 2)}, \quad m=1,2,3, \ldots,
$$

or more generally

$$
V_{k}(\eta, \tau)=-\frac{\partial}{\partial \eta}\left(\ln \sum_{m=0}^{k} a_{m} \varphi_{m}(\eta, \tau)\right)=-\frac{\sum_{m=1}^{k} m a_{m} H_{m-1}(\eta, \tau / 2)}{\sum_{m=0}^{k} a_{m} H_{m}(\eta, \tau / 2)}, \quad k=1,2,3, \ldots,
$$

where $a_{m}$ are arbitrary real constants. Therefore, we obtain that the variable coefficient FBE (3) with initial conditions $U_{m}\left(x, t_{0}\right)=-\left(m / \mu\left(t_{0}\right) x\right), \quad m=1,2,3, \ldots$, has rational solutions

$$
U_{m}(x, t)=\frac{\dot{r}(t)}{r(t)} \chi-\frac{m}{\mu(t)}\left(\frac{r\left(t_{0}\right)}{r(t)}\right)\left(\frac{H_{m-1}\left(\frac{r\left(t_{0}\right)}{r(t)} x, \frac{1}{2} \tau(t)\right)}{H_{m}\left(\frac{r\left(t_{0}\right)}{r(t)} x, \frac{1}{2} \tau(t)\right)}\right),
$$

and with more general initial conditions

$$
U_{k}\left(x, t_{0}\right)=-\frac{1}{\mu\left(t_{0}\right)}\left(\frac{\sum_{m=1}^{k} m a_{m} x^{m-1}}{\sum_{m=0}^{k} a_{m} x^{m}}\right), \quad k=1,2,3, \ldots
$$

it has rational solutions of the form

$$
U_{k}(x, t)=\frac{\dot{r}(t)}{r(t)} x-\frac{1}{\mu(t)}\left(\frac{r\left(t_{0}\right)}{r(t)}\right)\left(\frac{\sum_{m=1}^{k} m a_{m} H_{m-1}\left(\frac{r\left(t_{0}\right)}{r(t)} x, \frac{1}{2} \tau(t)\right)}{\sum_{m=0}^{k} a_{m} H_{m}\left(\frac{r\left(t_{0}\right)}{r(t)} x, \frac{1}{2} \tau(t)\right)}\right) .
$$

Solutions (20) and (21) can be written also in terms of standard Hermite polynomials $H_{m}(y)$ using that

$$
H_{m}(\eta, \tau / 2)=\frac{(\sqrt{\tau / 2})^{m}}{i^{m}} H_{m}\left(\frac{i \eta}{2 \sqrt{\tau / 2}}\right), \quad H_{m}(\eta, 0)=\eta^{m}, \quad m=0,1,2, \ldots,
$$

where $H_{m}(y)$, are defined by $\exp \left[2 y \xi-\xi^{2}\right]=\sum_{m=0}^{\infty}\left(\xi^{m} / m !\right) H_{m}(y)$. Thus, the points where Kampè de Feriet polynomials vanish can be found in terms of the well known zeros of the Hermite polynomials. For this, we denote by $y_{m}^{(l)}, l=1,2, \ldots, m$, the zeros of the Hermite polynomial $H_{m}(y)$, so that for each fixed $m$, one has $H_{m}\left(y_{m}^{(l)}\right)=0$ for all $l=1,2, \ldots, m$. From relation (22) it follows that 


$$
H_{m}(\eta, \tau / 2)=0 \Longleftrightarrow \eta=-i 2 y_{m}^{(l)} \sqrt{\tau / 2}, \quad l=1,2, \ldots, m .
$$

Thus, $U_{m}(x, t)$ given by (20) has singularities at points where $H_{m}\left(\left(r\left(t_{0}\right) / r(t)\right) x, \tau(t) / 2\right)=0$, and according to (23), the motion of these pole singularities is described by

$$
x_{m}^{(l)}(t)=-i 2 y_{m}^{(l)} \frac{r(t)}{r\left(t_{0}\right)} \sqrt{\frac{\tau(t)}{2}}, \quad l=1,2, \ldots, m .
$$

We note that, for a real-valued solution $r(t)$ and $\tau(t)>0, t \in I$, the solution $U_{m}(x, t)$ does not have moving singularities on the real line. It may have real singularity only at $x=0$. On the other hand, for some special choice of the coefficients $a_{m}$, the solutions $U_{k}(x, t)$ of the form (21) may have singularities moving on the real line. Illustrative examples are given in next section.

\section{Exactly solvable Burgers models}

\subsection{Forced Burgers equations with constant coefficients}

Burgers equation with constant coefficients and a forcing term linear in the space variable $x$

$$
U_{t}+U U_{x}=\frac{1}{2} U_{x x}-\omega_{0}^{2} x
$$

is a known integrable model and one can see for example [17,23]. For this model, according to Proposition 2.1 one has $\mu(t)=1, \gamma=0$, and $\omega^{2}(t)=\omega_{0}^{2}$ - real constant, so that the corresponding IVP for the second order linear ODE is

$$
\ddot{r}(t)+\omega_{0}^{2} r(t)=0, \quad r(0)=r_{0} \neq 0, \quad \dot{r}(0)=0 .
$$

When, $\omega_{0}^{2}=0$, one has $r(t)=r_{0}, \eta=x, \tau=t$, and formula (4) gives $U(x, t)=V(x, t)$, which is a solution of the standard Burgers equation, as expected. Using the approach in previous section, we will recall some particular solutions for the cases when $\omega_{0}^{2}>0$ and $\omega_{0}^{2}<0$.

\subsubsection{Case $\omega_{0}^{2}>0$}

In that case the IVP (26) has oscillating solution $r(t)=r_{0} \cos \left(\omega_{0} t\right)$ and the auxiliary function is $\tau(t)=\tan \left(\omega_{0} t\right) / \omega_{0}$. From Proposition 2.1, it follows that the forced BE (25) has solutions

$$
U(x, t)=-\omega_{0} \tan \left(\omega_{0} t\right) x+\sec \left(\omega_{0} t\right) V\left(\sec \left(\omega_{0} t\right) x, \tan \left(\omega_{0} t\right) / \omega_{0}\right),
$$

where $V(\eta, \tau)$ is a solution of the standard Burgers equation. In what follows, using the discussion in previous section, we will write explicitly some special solutions of BE (25), and note that, in the limit case $\omega_{0} \rightarrow 0$, these solutions $U(x, t)$ approach the solutions $V(x, t)$ of the standard BE.

(a) Forced Burgers Eq. (25) with initial condition $U(x, 0)=-A \tanh (A x),-\infty<x<\infty$, has shock type static wave solution

$$
U(x, t)=-\omega_{0} \tan \left(\omega_{0} t\right) x-A \sec \left(\omega_{0} t\right) \tanh \left(A \sec \left(\omega_{0} t\right) x\right),
$$

and with initial condition $U(x, 0)=c-A \tanh \left[A x+c_{0}\right], \infty<x<\infty$, it has shock wave solution of the form

$$
U(x, t)=-\omega_{0} \tan \left(\omega_{0} t\right) x+\sec \left(\omega_{0} t\right)\left(c-A \tanh \left[A\left(\sec \left(\omega_{0} t\right) x-\frac{c}{\omega_{0}} \tan \left(\omega_{0} t\right)\right)+c_{0}\right]\right) .
$$

In Fig. 1a, we plot solution (28), and in Fig. 1b the solution (29). Note that, due to the trigonometric functions in $U(x, t)$, finite time singularities appear periodically, and they can be interpreted as formation of shock discontinuities.

(b) Forced BE (25) has triangular wave solution

$$
U=-\omega_{0} \tan \left(\omega_{0} t\right) x+\sec \left(\omega_{0} t\right) \sqrt{\frac{\omega_{0}}{2 \pi \tan \left(\omega_{0} t\right)}}\left(\frac{\left(e^{A}-1\right) \exp \left[-\frac{\left(\sec \left(\omega_{0} t\right) x\right)^{2}}{2 \tan \left(\omega_{0} t\right) / \omega_{0}}\right]}{1+\frac{1}{2}\left(e^{A}-1\right) \operatorname{erfc}\left[\frac{\sec \left(\omega_{0} t\right) x}{\sqrt{2 \tan \left(\omega_{0} t\right) / \omega_{0}}}\right]}\right),
$$

for $t \in\left(0, \pi / 2 \omega_{0}\right)$. In general, triangular wave exists on time interval where $\tau(t)=\tan \left(\omega_{0} t\right) / \omega_{0}>0$.

(c) $N$-wave solution can also be considered for BE (25) using formula (17) on time interval where $\tau(t)>0$.

(d) Forced BE (25) subject to initial conditions $U_{m}(x, 0)=-m / x, m=1,2,3, \ldots$, has rational function solutions of the form

$$
U_{m}(x, t)=-\omega_{0} \tan \left(\omega_{0} t\right) x-\sec \left(\omega_{0} t\right)\left[\frac{m H_{m-1}\left(\sec \left(\omega_{0} t\right) x, \tan \left(\omega_{0} t\right) / 2 \omega_{0}\right)}{H_{m}\left(\sec \left(\omega_{0} t\right) x, \tan \left(\omega_{0} t\right) / 2 \omega_{0}\right)}\right],
$$

with moving pole singularities $x_{m}^{(l)}(t)=-i y_{m}^{l} \cos \left(\omega_{0} t\right) \sqrt{2 \tan \left(\omega_{0} t\right) / \omega_{0}}, l=1,2, \ldots, m$. 


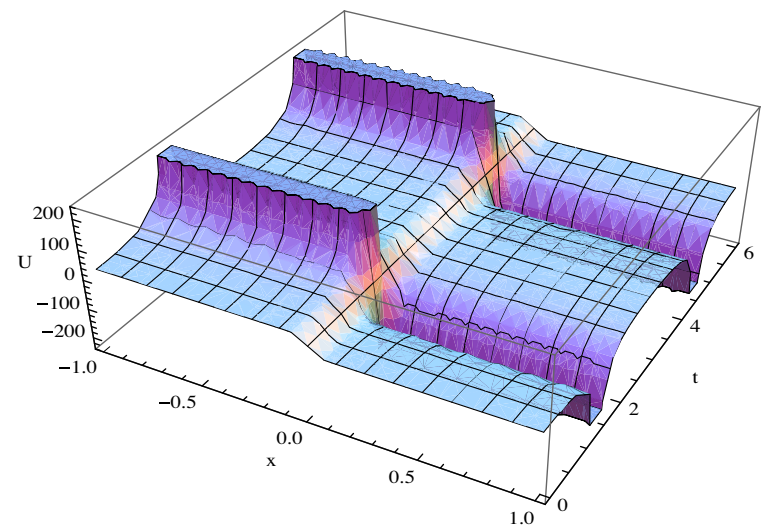

(a)

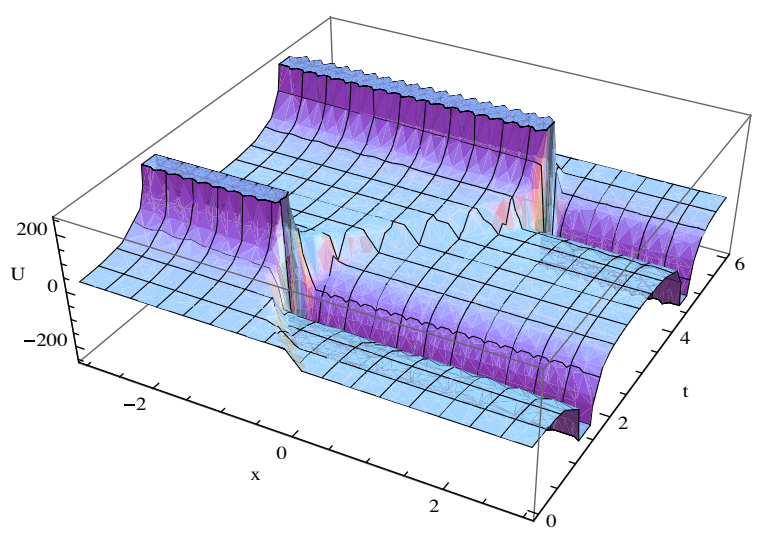

(b)

Fig. 1. (a) Shock type static wave solution $U(x, t)$ given by (28), $A=30, \omega_{0}=1$. (b) Shock wave solution $U(x, t)$ given by $(29), A=30, \omega_{0}=1, c=-1, c_{0}=0$.

\subsubsection{Case $\omega_{0}^{2}<0$}

When $\omega_{0}^{2}=-\tilde{\omega}^{2}, \tilde{\omega}>0$, the BE (25) becomes

$$
U_{t}+U U_{x}=\frac{1}{2} U_{x x}+\tilde{\omega}^{2} x
$$

and the corresponding $\operatorname{ODE}(26)$ has solution $r(t)=r_{0} \cosh (\tilde{\omega} t)$, where the auxiliary function is $\tau(t)=\tanh (\tilde{\omega} t) / \tilde{\omega}$. Therefore, solutions of BE (30) are of the form

$$
U(x, t)=\tilde{\omega} \tanh (\tilde{\omega} t) x+\frac{1}{\cosh (\tilde{\omega} t)} V\left(\frac{x}{\cosh (\tilde{\omega} t)}, \frac{\tanh (\tilde{\omega} t)}{\tilde{\omega}}\right),
$$

where $V(\eta, \tau)$ is a solution of the standard BE. As in the previous case, we see that in the limit $\tilde{\omega} \rightarrow 0$, the following particular exact solutions $U(x, t)$ approach the corresponding solution $V(x, t)$ of the standard BE.

(a) The forced BE (30) with initial condition $U(x, 0)=c-A \tanh [A x],-\infty<x<\infty$ has one-shock wave solution

$$
U(x, t)=\tilde{\omega} \tanh (\tilde{\omega} t) x+\frac{1}{\cosh (\tilde{\omega} t)}\left(c-A \tanh \left[\frac{A}{\cosh (\tilde{\omega} t)}\left(x-c \frac{\sinh (\tilde{\omega} t)}{\tilde{\omega}}\right)\right]\right),
$$

which amplitude depends on time, the center of the wave profile moves according to $x(t)=c \sinh (\tilde{\omega} t) / \tilde{\omega}$, and its velocity is $v(t)=c \cosh (\tilde{\omega} t)$. In Fig. 2a, we plot one-shock wave and observe that the shock center moves with increasing velocity

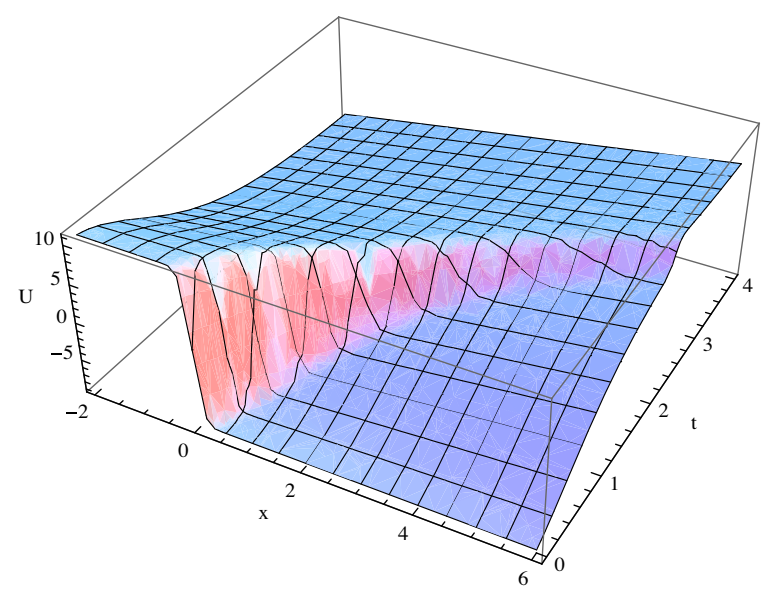

(a)

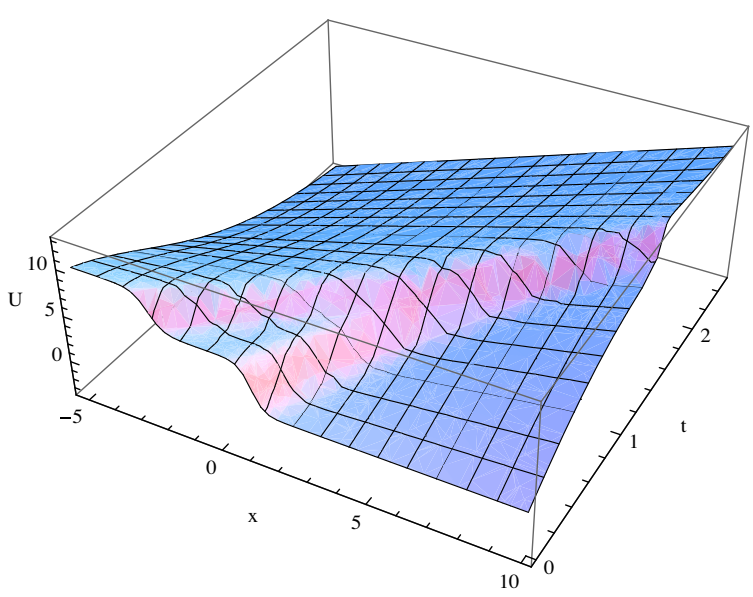

(b)

Fig. 2. (a) One-shock wave solution $U(x, t)$ given by (32), $A=15, c=1, \tilde{\omega}=1$. (b) Two-shock wave solution $U(x, t)$ given by (33), $a_{1}=1, a_{2}=6$, $a_{3}=10, p_{1}^{0}=0, p_{2}^{0}=5, p_{3}^{0}=-5, \tilde{\omega}=1$. 
$v(t)=\cosh (t), t>0$, and its amplitude decays with time. Also, one can see that the initial profile is localized in the sense that $U(x, 0)$ approaches constant as $x \rightarrow \pm \infty$, while $U(x, t) \rightarrow \tilde{\omega} x$ when $t \rightarrow \infty$.

In general, BE (30) has multi-shock type solutions of the form

$$
U(x, t)=\tilde{\omega} \tanh (\tilde{\omega} t) x+\frac{1}{\cosh (\tilde{\omega} t)}\left[\frac{a_{1} \exp \left[p_{1}(x, t)\right]+a_{2} \exp \left[p_{2}(x, t)\right]+\cdots+a_{k} \exp \left[p_{k}(x, t)\right]}{\exp \left[p_{1}(x, t)\right]+\exp \left[p_{2}(x, t)\right]+\cdots+\exp \left[p_{k}(x, t)\right]}\right],
$$

where

$$
p_{i}(x, t)=-\frac{a_{i}}{\cosh (\tilde{\omega} t)} x+\frac{a_{i}^{2}}{2 \tilde{\omega}} \tanh (\tilde{\omega} t)+p_{i}^{0}, \quad i=1,2, \ldots
$$

In Fig. 2b, we illustrate the fusion of two-shock type solution.

(b) Triangular wave solution of $\mathrm{BE}(30)$ is

$$
U(x, t)=\tilde{\omega} \tanh (\tilde{\omega} t) x+\sqrt{\frac{\tilde{\omega}}{\pi \sinh (2 \tilde{\omega} t)}}\left(\frac{\left(e^{A}-1\right) \exp \left[-\frac{\tilde{\omega}}{\sinh (2 \tilde{\omega} t)} x^{2}\right]}{1+\frac{1}{2}\left(e^{A}-1\right) \operatorname{erfc}\left[\sqrt{\frac{\tilde{\omega}}{\sinh (2 \tilde{\omega} t)}} x\right]}\right),
$$

which corresponds to Dirac-delta initial distribution, see Fig. 3a.

(c) $\mathrm{N}$-wave solution of $\mathrm{BE}(30)$ is of the form

$$
U(x, t)=\tilde{\omega} \tanh (\tilde{\omega} t) x+\frac{2 \tilde{\omega} x}{\sinh (2 \tilde{\omega} t)}\left[\frac{\sqrt{\frac{a \tilde{\omega}}{\tanh (\tilde{\omega} t)}} \exp \left[-\frac{\tilde{\omega}}{\sinh (2 \tilde{\omega} t)} x^{2}\right]}{1+\sqrt{\frac{a \tilde{\omega}}{\tanh (\tilde{\omega} t)}} \exp \left[-\frac{\tilde{\omega}}{\sinh (2 \tilde{\omega} t)} x^{2}\right]}\right]
$$

and one can see Fig. 3b.

(d) Rational function solutions of $\mathrm{BE}$ (30) subject to initial conditions $U_{m}(x, 0)=-m / x, m=1,2,3, \ldots$, are of the form

$$
U_{m}(x, t)=\tilde{\omega} \tanh (\tilde{\omega} t) x+\frac{1}{\cosh (\tilde{\omega} t)}\left(\frac{m H_{m-1}(x / \cosh (\tilde{\omega} t), \tanh (\tilde{\omega} t) / 2 \tilde{\omega})}{H_{m}(x / \cosh (\tilde{\omega} t), \tanh (\tilde{\omega} t) / 2 \tilde{\omega})}\right)
$$

For each fixed $m$, the rational solution (35) has pole singularities whose motion in the complex plane is described by

$$
x_{m}^{(l)}(t)=-i y_{m}^{(l)} \sqrt{\frac{\sinh (2 \tilde{\omega} t)}{\tilde{\omega}}}, \quad t>0, l=1,2, \ldots, m,
$$

and as $\tilde{\omega} \rightarrow 0$ they approach the well known poles $x_{m}^{(l)}(t)=-i y_{m}^{(l)} \sqrt{2 t}$ of the standard BE.

Finally, we note that for the above particular solutions, one has $\lim _{t \rightarrow \infty} U(x, t)=\tilde{\omega} x$. Similar result was obtained in [22], where the long-time asymptotics for solutions of the BE (30) were discussed according to the properties of the initial profile.

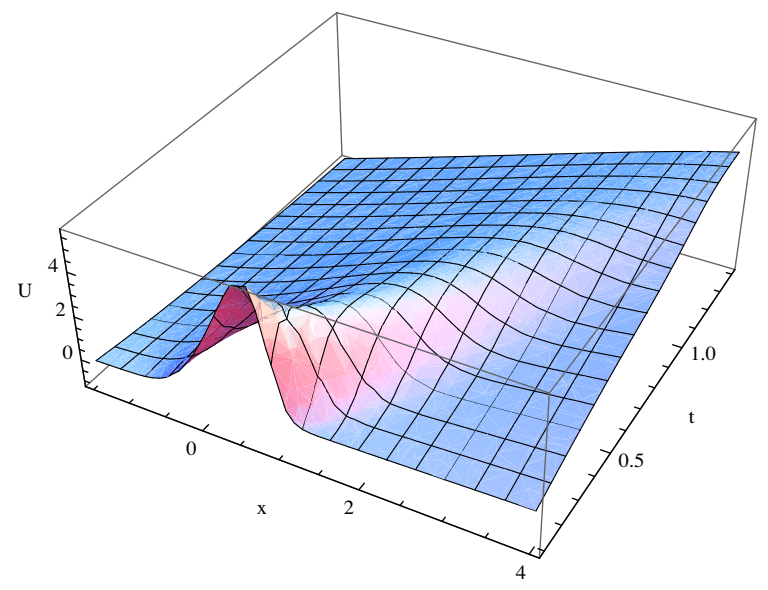

(a)

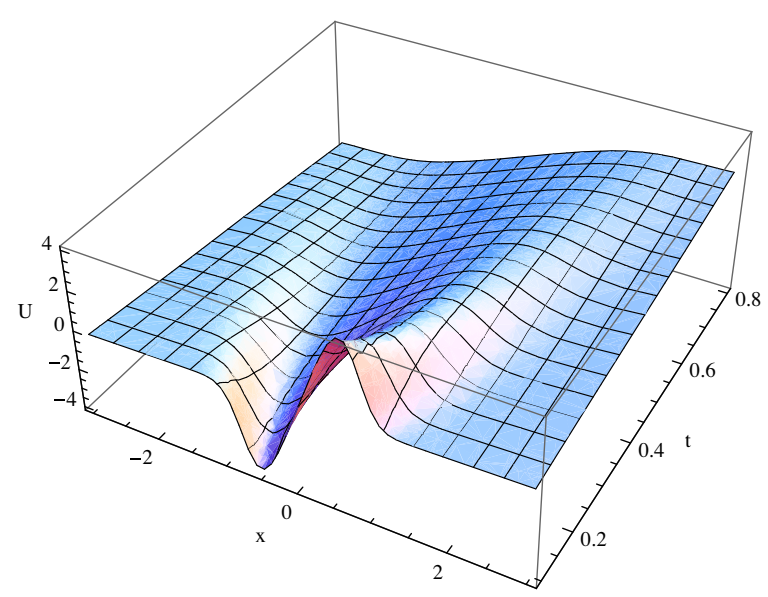

(b)

Fig. 3. (a) Triangular wave solution $U(x, t)$ on time interval $0.1<t<1$, with $A=50, \tilde{\omega}=1$. (b) $N$-wave solution (34) on time interval $0.1<t<0.8, a=15, \tilde{\omega}=1$. 
3.2. Forced Burgers equations with constant damping and exponentially decaying diffusion coefficient

In this part, we consider exactly solvable forced Burgers equations

$$
U_{t}+\gamma U+U U_{x}=\frac{1}{2} e^{-\gamma t} U_{x x}-\omega_{0}^{2} x, \quad-\infty<x<\infty, t>0,
$$

with constant damping $\Gamma(t)=\gamma>0$, exponentially decaying diffusion coefficient $D(t)=e^{-\gamma t} / 2, \omega^{2}(t)=\omega_{0}^{2}>0$ and $\mu(t)=e^{\gamma t}$. The corresponding IVP for the linear ODE is then

$$
\ddot{r}+\gamma \dot{r}+\omega_{0}^{2} r=0, \quad r(0)=r_{0} \neq 0, \dot{r}(0)=0,
$$

and it has three different type of solutions depending on the sign of $\omega_{0}^{2}-\left(\gamma^{2} / 4\right)$. In what follows, for each case we discuss separately the related variable coefficient Burgers equations (36).

\subsubsection{Critical damping case}

If $\omega_{0}^{2}-\left(\gamma^{2} / 4\right)=0$, the IVP (37) for the linear ODE, has solution

$$
r_{1}(t)=r_{1}(0) e^{-\frac{\gamma t}{2}}\left(1+\frac{\gamma}{2} t\right)
$$

and the auxiliary function is $\tau_{1}(t)=t /(1+\gamma t / 2)$. Therefore, the $B E$ (36) has solutions of the form

$$
U(x, t)=-\left(\frac{\gamma}{2}\right)^{2}\left(\frac{t}{1+\frac{\gamma}{2} t} x\right)+\left(\frac{e^{-\gamma t / 2}}{1+\frac{\gamma}{2} t}\right) V\left(\frac{e^{\gamma t / 2} x}{1+\frac{\gamma}{2} t}, \frac{t}{1+\frac{\gamma}{2} t}\right),
$$

where $V(\eta, \tau)$ satisfies the standard BE.

Clearly, when $\omega_{0} \rightarrow 0$, one has also $\gamma \rightarrow 0$, and in that case we can see that the following particular solutions $U(x, t)$ approach solutions $V(x, t)$ of the standard BE.

(a) $\mathrm{BE}(36)$ with initial condition $U(x, 0)=c-A \tanh [A x]$ has shock wave solution

$$
U(x, t)=-\left(\frac{\gamma}{2}\right)^{2}\left(\frac{t}{1+\frac{\gamma}{2} t}\right) x+\left(\frac{e^{-\gamma t / 2}}{1+\frac{\gamma}{2} t}\right)\left(c-A \tanh \left[A\left(\frac{e^{\gamma t / 2}}{1+\frac{\gamma}{2} t}\left(x-c t e^{-\gamma t / 2}\right)\right)\right],\right.
$$

which shock amplitude decays with time eventually going to zero, and its "center" moves with velocity $v(t)=c(1-\gamma t / 2) e^{-\gamma t / 2}$, see Fig. 4. Multi-shock wave solutions of the BE (36), can be found from the general solution

$$
U(x, t)=-\left(\frac{\gamma}{2}\right)^{2}\left(\frac{t}{1+\frac{\gamma}{2} t}\right) x+\left(\frac{e^{-\gamma t / 2}}{1+\frac{\gamma}{2} t}\right)\left[\frac{a_{1} \exp \left[p_{1}(x, t)\right]+\cdots+a_{k} \exp \left[p_{k}(x, t)\right]}{\exp \left[p_{1}(x, t)\right]+\cdots+\exp \left[p_{k}(x, t)\right]}\right],
$$

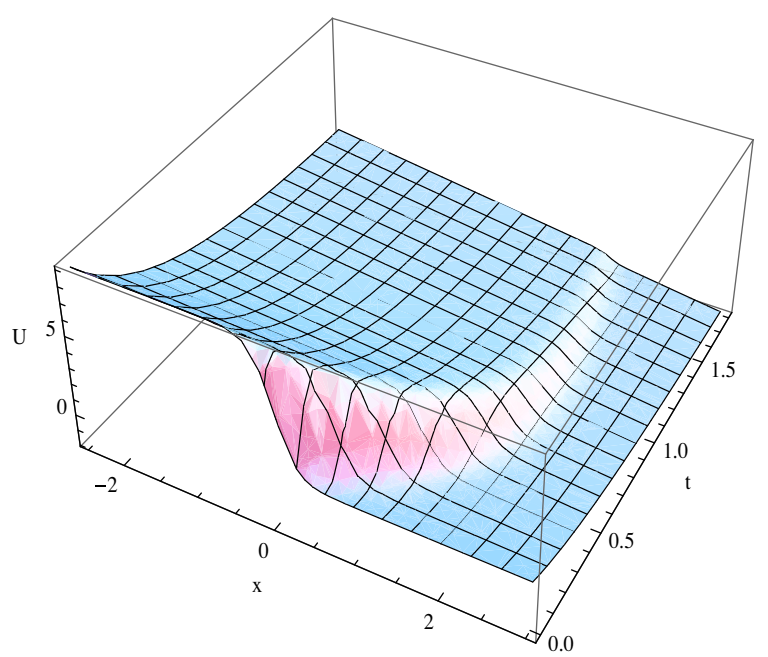

(a)

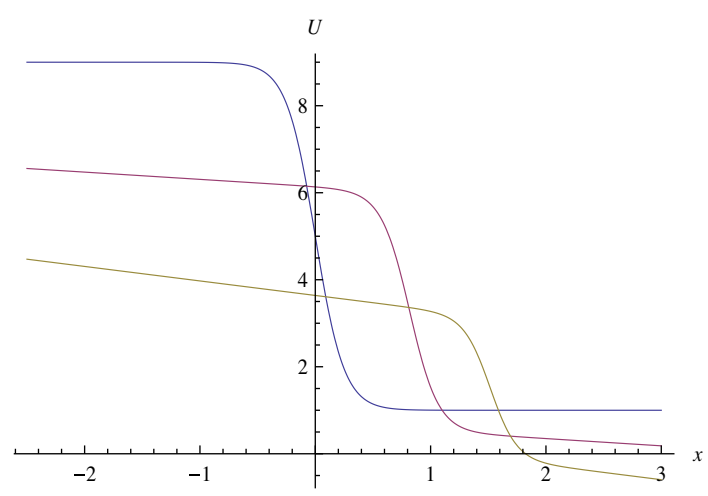

(b)

Fig. 4. Critical damping case. (a) One-shock wave solution $U(x, t)$ given by $(40), \gamma=2, A=4, c=5, c_{0}=0$. (b) The profiles of $U(x, t)$ at times $t=0, t=0.2, t=0.4$. 
where

$$
p_{i}(x, t)=-a_{i}\left(\frac{e^{\gamma t / 2}}{1+\frac{\gamma}{2} t}\right) x+\frac{a_{i}^{2}}{2}\left(\frac{t}{1+\frac{\gamma}{2} t}\right)+p_{i}^{0}, \quad i=1,2, \ldots, k,
$$

and $a_{i}, p_{i}^{0}$ are real constants. In Fig. 5 we plot two-shock wave solution $U(x, t)$, with special choices in (41), $k=3, \gamma=2, a_{1}=1, p_{1}^{0}=0, a_{2}=7, p_{2}^{0}=5, a_{3}=15, p_{3}^{0}=-4$. We observe fusion of the two-shock wave, which shock contribution eventually goes to zero.

(b) Using again the results in Section 2, one obtains that the BE (36) has generalized triangular wave solution

$$
U(x, t)=-\left(\frac{\gamma}{2}\right)^{2}\left(\frac{t}{\left(1+\frac{\gamma}{2} t\right)}\right) x-\frac{e^{-\frac{\gamma}{2} t}}{\left(1+\frac{\gamma}{2} t\right)} \sqrt{\frac{(1+\gamma t / 2)}{2 \pi t}}\left(\frac{\left(e^{A}-1\right) \exp \left[-e^{\gamma t} x^{2} / 2 t(1+\gamma t / 2)\right]}{1+\frac{1}{2}\left(e^{A}-1\right) \operatorname{erfc}\left[e^{\gamma t / 2} x / \sqrt{2 t(1+\gamma t / 2)}\right]}\right),
$$

corresponding to Dirac-delta initial profile, see Fig. 6.

(c) BE (36) for the critical damping case has $N$-wave solution of the form (17) with $r(t)=r_{1}(t)$ and $\tau(t)=\tau_{1}(t)$. Explicit form of this generalized $N$-wave solution is

$$
U(x, t)=-\left(\frac{\gamma}{2}\right)^{2}\left(\frac{t}{\left(1+\frac{\gamma}{2} t\right)}\right) x+\frac{x}{t\left(1+\frac{\gamma}{2} t\right)}\left(\frac{\sqrt{\frac{a(1+\gamma t / 2)}{t}} \exp \left[-e^{\gamma t} x^{2} / 2 t(1+\gamma t / 2)\right]}{1+\sqrt{\frac{a(1+\gamma t / 2)}{t}} \exp \left[-e^{\gamma t} x^{2} / 2 t(1+\gamma t / 2)\right]}\right),
$$

and one can see Fig. 7.

(d) Rational solutions of the $\mathrm{BE}(36)$ with initial conditions $U_{m}(x, 0)=-m(1 / x), m=1,2, \ldots$ are of the form

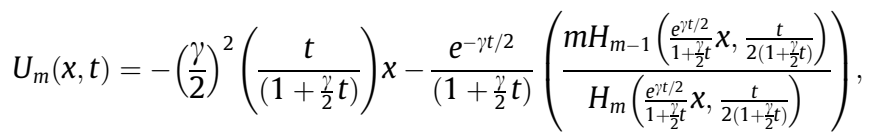

and their behavior for $m=1$ and $m=2$ is illustrated in Fig. 8. According to (24), for each $m$, the motion of the pole singularities is described by

$$
x_{m}^{(l)}(t)=-i \sqrt{2} y_{m}^{(l)} e^{-\gamma t / 2} \sqrt{t(1+\gamma t / 2)}, \quad l=1,2, \ldots
$$

Since $\gamma>0$ and $t>0$, clearly $U_{m}(x, t)$ has no moving singularities on the real line. On the other hand, forced BE (36) with more general initial conditions

$$
U_{k}(x, 0)=-\left(\sum_{m=1}^{k} m a_{m} x^{m-1}\right) / \sum_{m=0}^{k} a_{m} x^{m}, \quad k=1,2, \ldots,
$$

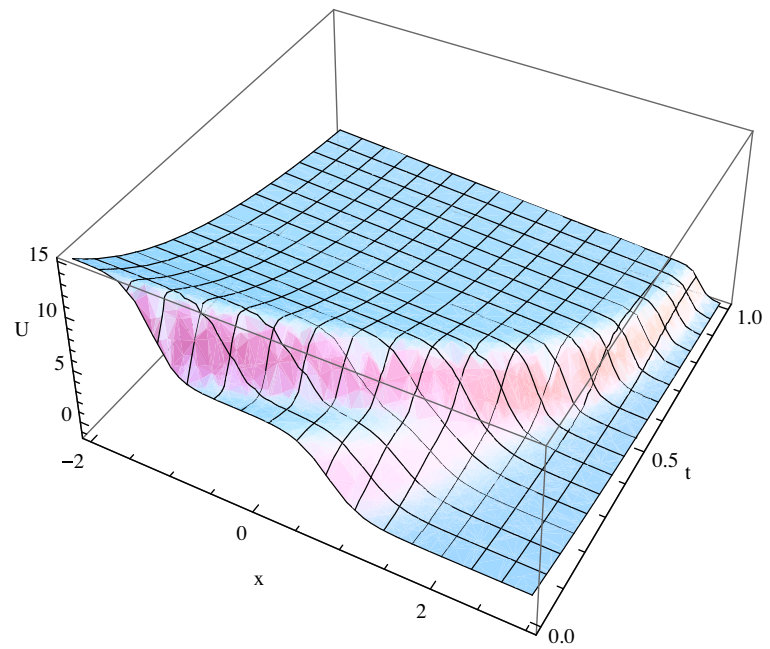

(a)

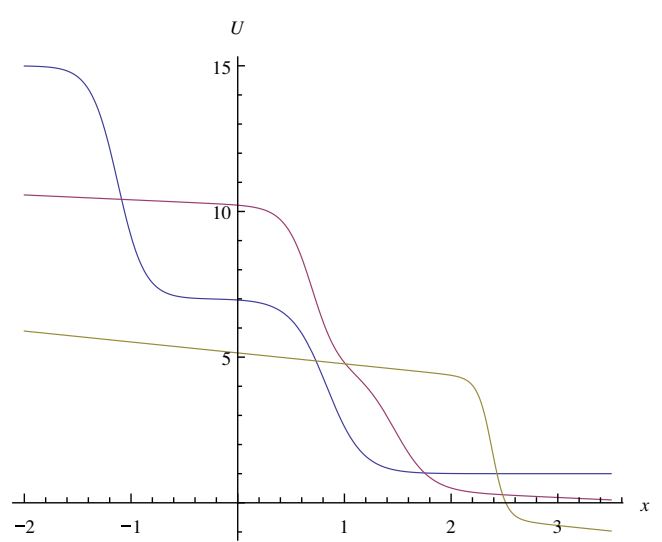

(b)

Fig. 5. Critical damping case. (a) Fusion of the two-shock wave $U(x, t)$. (b) Profiles of $U(x, t)$ at times $t=0, t=0.2$, and $t=0.6$. 


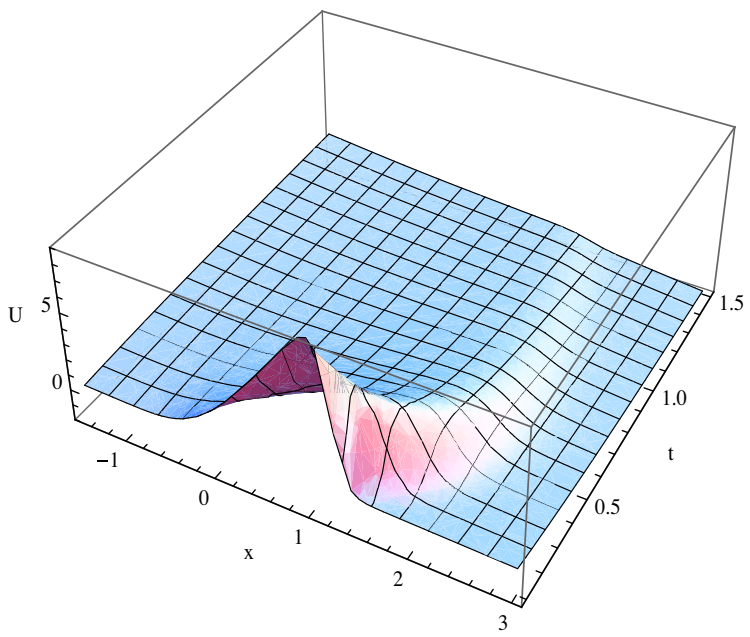

(a)

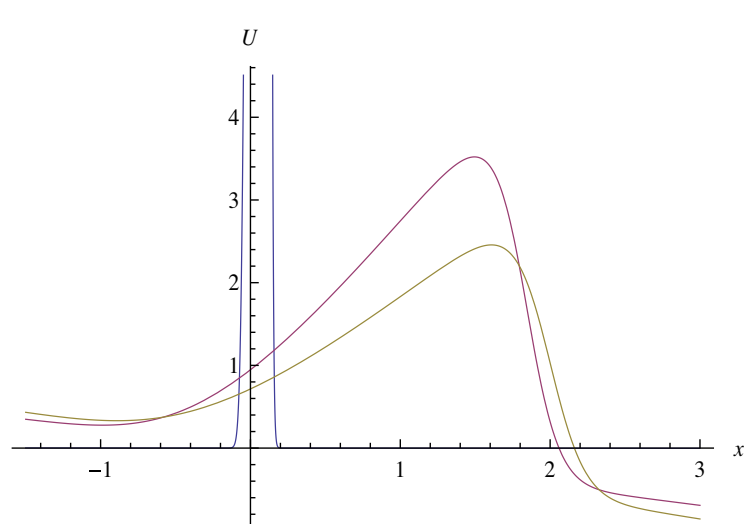

(b)

Fig. 6. Critical damping case. (a) Plot of triangular wave solution $U(x, t)$ for time interval $0.1<t<1.5$, and $A=10, \gamma=2$. (b) Profile of $U(x, t)$ at times $t=0.001, t=0.3$, and $t=0.4$.

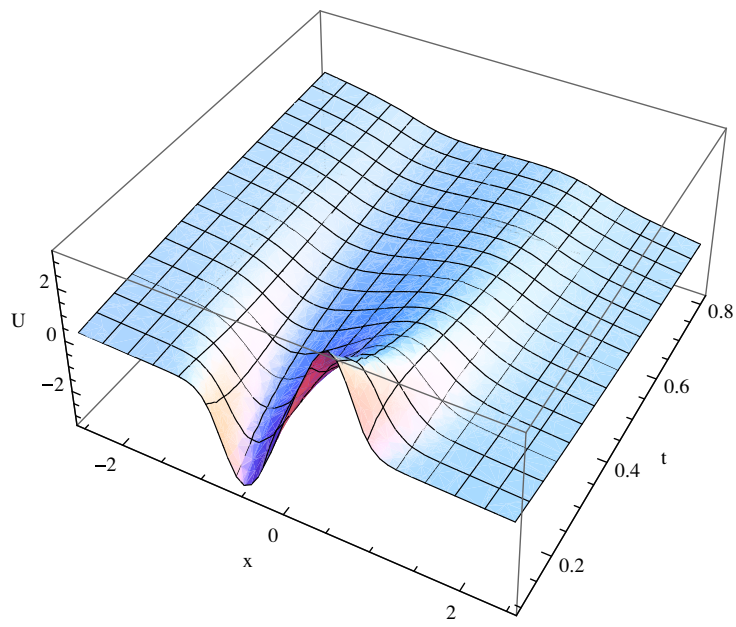

(a)

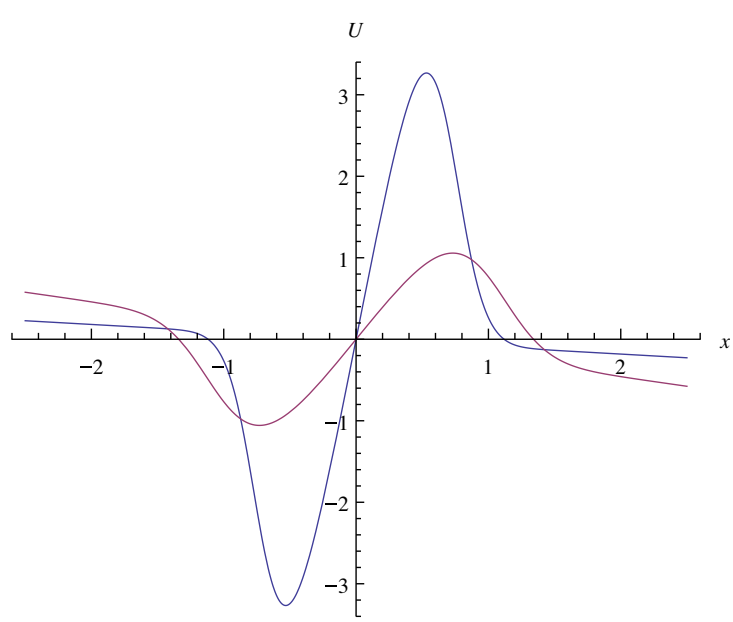

(b)

Fig. 7. Critical damping case. (a) Plot of $N$-wave solution $U(x, t)$ for time interval $0.1<t<0.8$ with $a=10$ and $\gamma=2$. (b) Profiles of $U(x, t)$ at times $t=0.01, t=0.3$.

has the following rational solutions

$$
U_{k}(x, t)=-\left(\frac{\gamma}{2}\right)^{2}\left(\frac{t}{\left(1+\frac{\gamma}{2} t\right)}\right) x-\frac{e^{-\gamma t / 2}}{\left(1+\frac{\gamma}{2} t\right)}\left(\frac{\sum_{m=1}^{k} m a_{m} H_{m-1}\left(\frac{e^{\gamma} t / 2}{1+\frac{\gamma}{2} t} x, \frac{t}{2\left(1+\frac{\gamma}{2} t\right)}\right)}{\sum_{m=0}^{k} a_{m} H_{m}\left(\frac{e \gamma t / 2}{1+\frac{\gamma}{2} t} x, \frac{t}{2\left(1+\frac{\gamma}{2} t\right)}\right)}\right) .
$$

As an example, in Fig. 9 we consider solution $U_{2}(x, t)$, which has one fixed singularity $x=0$ and one moving singularity on the real line approaching $x=0$ as $t \rightarrow \infty$.

At the end of this section, we note that for the above particular solutions one has $\lim _{t \rightarrow \infty} U(x, t)=-(\gamma / 2) x$, so that in the long-time limit the system becomes stable with velocity proportional to the displacement. Here, the limit function $-(\gamma / 2) x$ is also a stationary solution for the BE (36). 


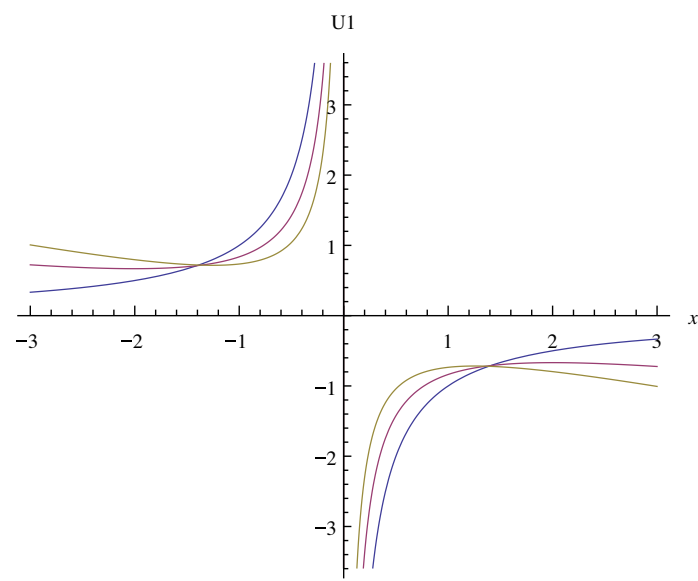

(a)

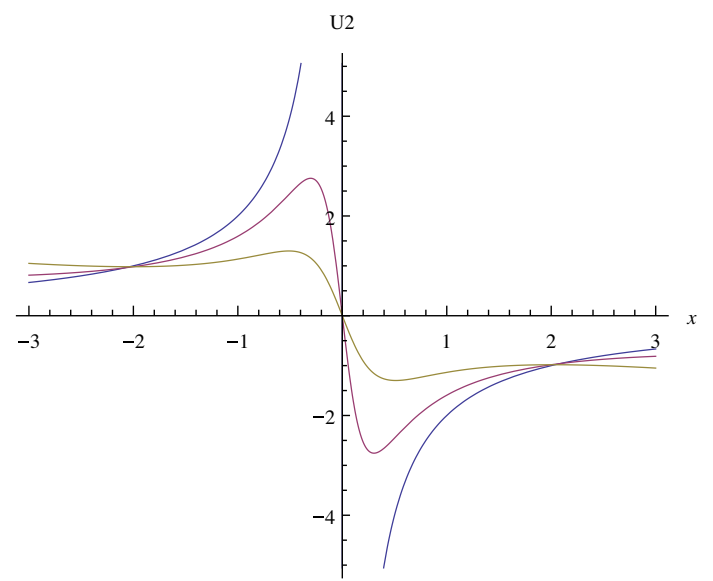

(b)

Fig. 8. (a) Profiles of the rational solution $U_{1}(x, t)$ given by (42) for $t=0, t=0.2, t=0.4$. b) Profiles of the rational solution $U_{2}(x, t)$ given by $(42)$ for $t=0, t=0.1, t=0.3,(\gamma=2)$.

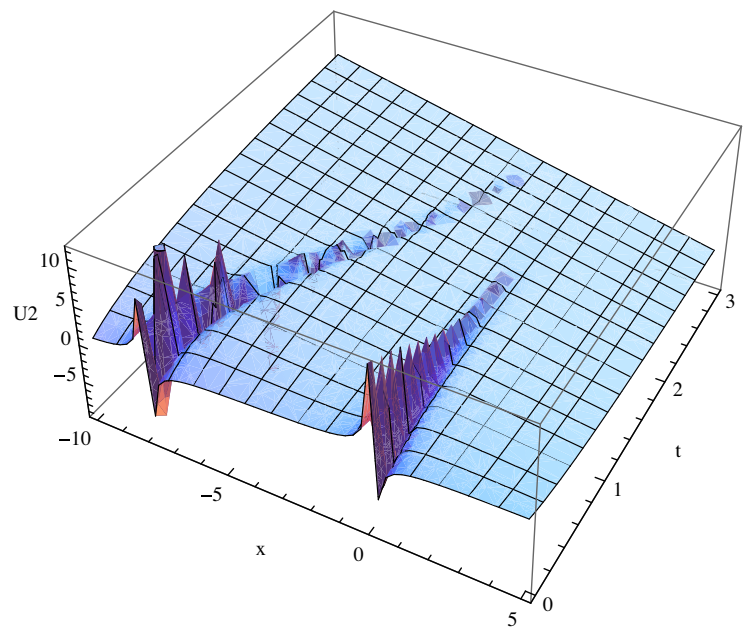

(a)

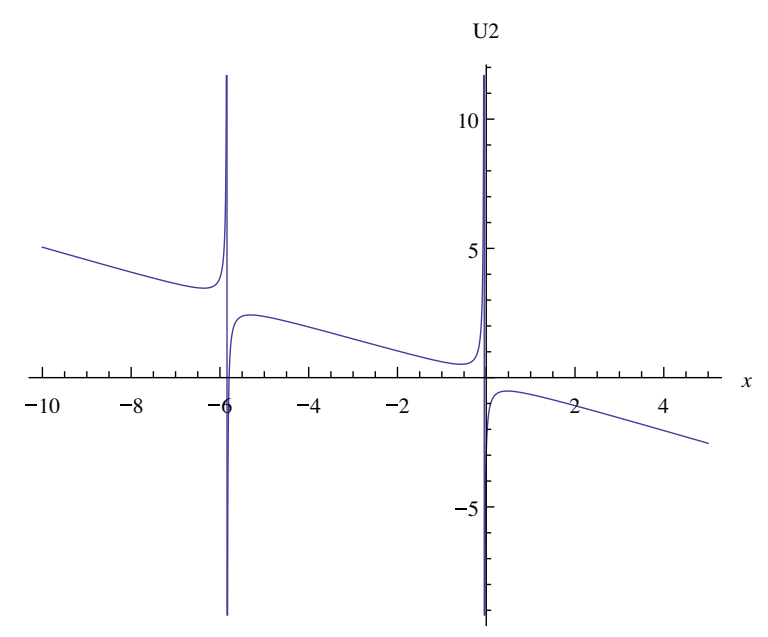

(b)

Fig. 9. (a) Rational solution $U_{2}(x, t)$ of the form (44), $\gamma=2, a_{0}=0, a_{1}=8, a_{2}=1$. (b) The profile corresponds to time $t=1$.

\subsubsection{Under damping case}

If $\omega_{0}^{2}-\left(\gamma^{2} / 4\right)>0$, then the ODE (37) has oscillating solution

$$
r_{2}(t)=r_{2}(0) \frac{\omega_{0}}{\Omega} e^{-\frac{\gamma t}{2}} \cos [\Omega t-\alpha]
$$

where $\Omega=\sqrt{\omega_{0}^{2}-\left(\gamma^{2} / 4\right)}>0, \alpha=\tan ^{-1}\left(\frac{\gamma}{2 \Omega}\right)$, and the auxiliary function is

$$
\tau_{2}(t)=\frac{\Omega}{\omega_{0}^{2}}\left(\tan [\Omega t-\alpha]+\frac{\gamma}{2 \Omega}\right)
$$

Then, BE (36) has solutions of the form

$$
U(x, t)=-\left(\frac{\gamma}{2}+\Omega \tan [\Omega t-\alpha]\right) x+\frac{\Omega}{\omega_{0}} \frac{e^{-\gamma t / 2}}{\cos [\Omega t-\alpha]} V\left(\frac{\Omega}{\omega_{0}} \frac{e^{\gamma t / 2} x}{\cos [\Omega t-\alpha]}, \frac{\Omega}{\omega_{0}^{2}}\left(\tan [\Omega t-\alpha]+\frac{\gamma}{2 \Omega}\right)\right),
$$

where $V(\eta, \tau)$ satisfies the standard BE. 
When $\gamma \rightarrow 0$, one has $\Omega \rightarrow \omega_{0}>0$ and $\alpha \rightarrow 0$. In that case, it is not difficult to see that the bellow given solutions of the forced BE (36) with variable coefficients approach the corresponding solutions of the forced BE (25) with constant coefficients.

(a) BE (36) has shock type static solution

$$
U(x, t)=-\left(\frac{\gamma}{2}+|\Omega| \tan [\Omega t-\alpha]\right) x-\frac{A \Omega}{\omega_{0}} \frac{e^{-\gamma t / 2}}{\cos [\Omega t-\alpha]} \tanh \left[\frac{A \Omega}{\omega_{0}} \frac{e^{\gamma t / 2} x}{\cos [\Omega t-\alpha]}\right],
$$

which behavior is illustrated in Fig. 10a, and it has shock wave solution in the form

$$
\begin{aligned}
U(x, t)= & -\left(\frac{\gamma}{2}+\Omega \tan [\Omega t-\alpha]\right) x+\frac{\Omega}{\omega_{0}} \\
& \times \frac{e^{-\gamma t / 2}}{\cos [\Omega t-\alpha]}\left(c-A \tanh \left[A\left(\frac{\Omega}{\omega_{0}} \frac{e^{\gamma t / 2} x}{\cos [\Omega t-\alpha]}-c \frac{\Omega}{\omega_{0}^{2}}\left(\tan [\Omega t-\alpha]+\frac{\gamma}{2 \Omega}\right)\right)\right]\right),
\end{aligned}
$$

which for some particular parameters is plotted in Fig. 10b. Note that the solution wave (49) is broken by shock discontinuities which appear periodically at finite times.

Multi-shock wave solutions are found as

$$
U(x, t)=\frac{\dot{r}_{2}(t)}{r_{2}(t)} x+e^{-\gamma t} \frac{r_{2}\left(t_{0}\right)}{r_{2}(t)}\left[\frac{a_{1} \exp \left[p_{1}(x, t)\right]+a_{2} \exp \left[p_{2}(x, t)\right]+\cdots+a_{k} \exp \left[p_{k}(x, t)\right]}{\exp \left[p_{1}(x, t)\right]+\exp \left[p_{2}(x, t)\right]+\cdots+\exp \left[p_{k}(x, t)\right]}\right],
$$

where

$$
p_{i}(x, t)=-a_{i} \frac{r_{2}\left(t_{0}\right)}{r_{2}(t)} x+\frac{a_{i}^{2}}{2} \tau_{2}(t)+p_{i}^{0}, \quad i=1,2, \ldots, k,
$$

$r_{2}(t)$ is given by (45), and $\tau_{2}(t)$ is given by (46).

(b) BE (36) has generalized triangular wave solution of the form (16) with $r(t)=r_{2}(t)$ and $\tau(t)=\tau_{2}(t)$ on a time interval where $\tau_{2}(t)>0$.

(c) Generalized $N$-wave solution of BE (36) for the under damping case can be found using formula (17) on a time interval where $\tau_{2}(t)>0$.

(d) BE (36) with initial conditions

$$
U_{k}(x, 0)=-\left(\sum_{m=1}^{k} m a_{m} x^{m-1}\right) / \sum_{m=0}^{k} a_{m} x^{m}, \quad k=1,2, \ldots,
$$

has rational function solutions

$$
U_{k}(x, t)=\frac{\dot{r}_{2}(t)}{r_{2}(t)} x-e^{-\gamma t}\left(\frac{r_{2}(0)}{r_{2}(t)}\right)\left(\frac{\sum_{m=1}^{k} m a_{m} H_{m-1}\left(\frac{r_{2}(0)}{r_{2}(t)} x, \frac{1}{2} \tau_{2}(t)\right)}{\sum_{m=0}^{k} a_{m} H_{m}\left(\frac{r_{2}(0)}{r_{2}(t)} x, \frac{1}{2} \tau_{2}(t)\right)}\right) .
$$

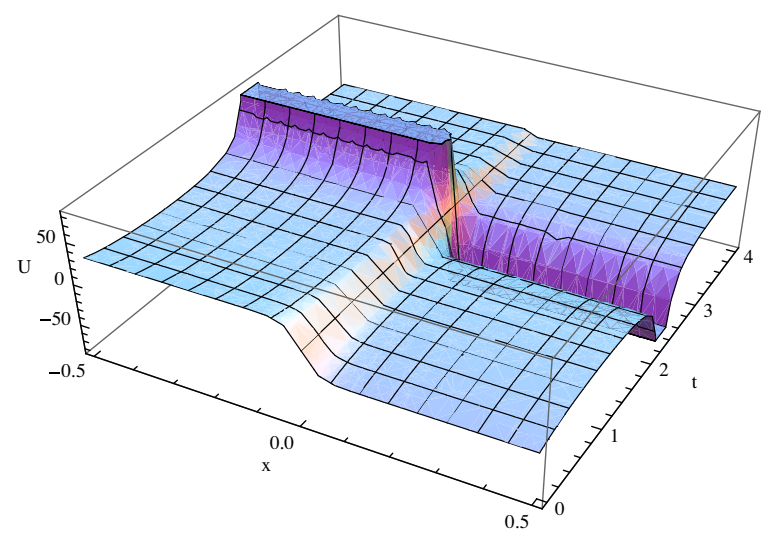

(a)

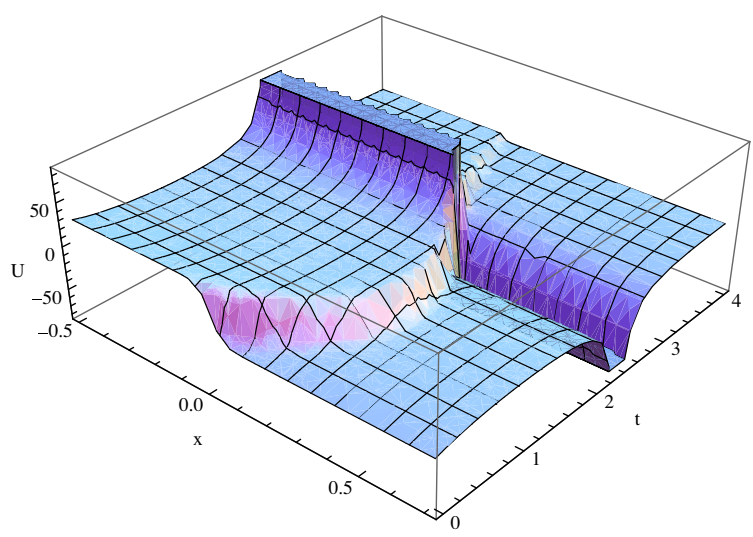

(b)

Fig. 10. Under damping case. (a) Shock type static solution $U(x, t)$ given by (48), $\omega_{0}=1, \gamma=1, A=30$. (b) Shock wave solution $U(x, t)$ given by (49), $\omega_{0}=1, \gamma=1, A=30, c=0.6$. 


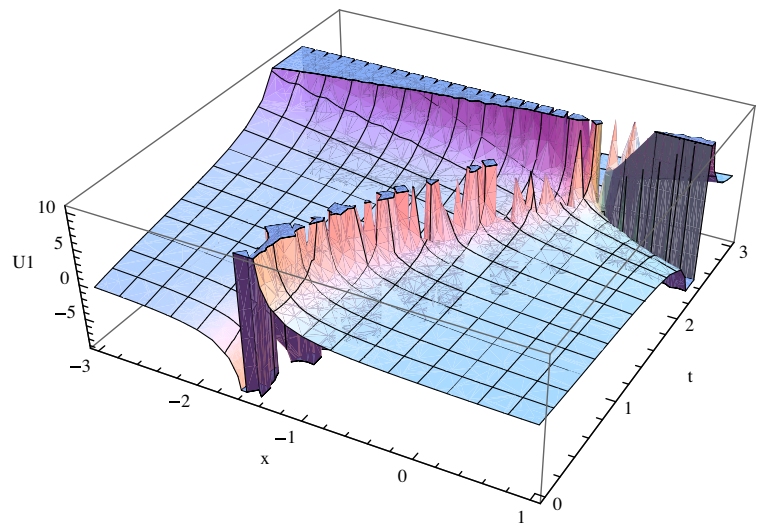

(a)

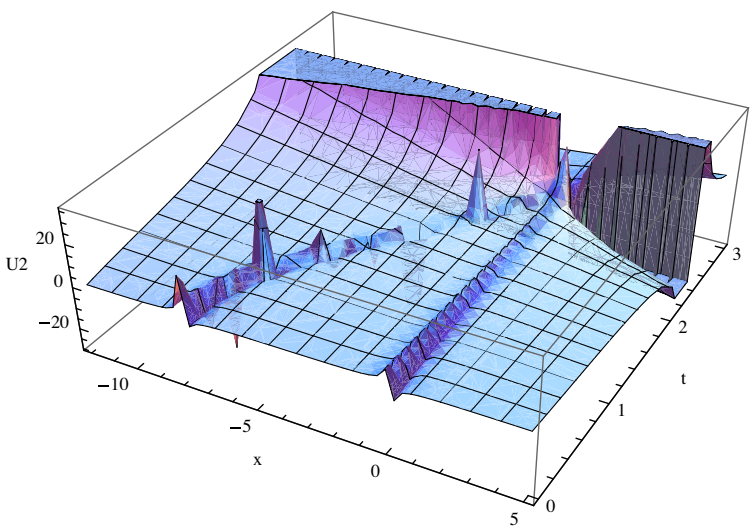

(b)

Fig. 11. Under damping case. (a) Rational solution $U_{1}(x, t), \omega_{0}=1, \gamma=1, a_{0}=1.5, a_{1}=1$. (b) Rational solution $U_{2}(x, t), \quad \omega_{0}=1, \gamma=1$, $a_{0}=0, a_{1}=8, a_{2}=1$.

In Fig. 11, we plot $U_{1}(x, t)$ and $U_{2}(x, t)$ for some particular choice of the coefficients. In both cases we observe moving singularities.

\subsubsection{Over damping case}

When $\omega_{0}^{2}-\left(\gamma^{2} / 4\right)<0$, the IVP (37) has solution

$$
r_{3}(t)=r_{3}(0) \frac{\omega_{0}}{\Omega^{\prime}} e^{-\frac{\gamma t}{2}} \sinh \left[\Omega^{\prime} t+\beta\right]
$$

where $\Omega^{\prime}=\sqrt{\left|\omega_{0}^{2}-\left(\gamma^{2} / 4\right)\right|}$, and $\beta=\operatorname{coth}^{-1}\left(\frac{\gamma}{2 \Omega^{\prime}}\right)$. Then,

$$
\tau_{3}(t)=-\frac{\Omega^{\prime}}{\omega_{0}^{2}}\left(\operatorname{coth}\left[\Omega^{\prime} t+\beta\right]-\frac{\gamma}{2 \Omega^{\prime}}\right),
$$

and thus BE (36) has solutions of the form

$$
\left.U(x, t)=\left(-\frac{\gamma}{2}+\Omega^{\prime} \operatorname{coth}\left[\Omega^{\prime} t+\beta\right]\right) x+\left(\frac{\Omega^{\prime} e^{-\gamma t / 2}}{\omega_{0} \sinh \left[\Omega^{\prime} t+\beta\right]}\right) V\left(\frac{\Omega^{\prime} e^{\gamma t / 2} x}{\omega_{0} \sinh \left[\Omega^{\prime} t+\beta\right]},-\frac{\Omega^{\prime}}{\omega_{0}^{2}}\left(\operatorname{coth}\left[\Omega^{\prime} t+\beta\right]-\frac{\gamma}{2 \Omega^{\prime}}\right)\right)\right)
$$

where $V(\eta, \tau)$ satisfies the standard BE (6).

(a) Shock and multi-shock wave solutions for the forced BE (36) can be obtained from the general expression

$$
U(x, t)=\frac{\dot{r}_{3}(t)}{r_{3}(t)} x+e^{-\gamma t} \frac{r_{3}\left(t_{0}\right)}{r_{3}(t)}\left[\frac{a_{1} \exp \left[p_{1}(x, t)\right]+a_{2} \exp \left[p_{2}(x, t)\right]+\cdots+a_{k} \exp \left[p_{k}(x, t)\right]}{\exp \left[p_{1}(x, t)\right]+\exp \left[p_{2}(x, t)\right]+\cdots+\exp \left[p_{k}(x, t)\right]}\right],
$$

where

$$
p_{i}(x, t)=-a_{i} \frac{r_{3}\left(t_{0}\right)}{r_{3}(t)} x+\frac{a_{i}^{2}}{2} \tau_{3}(t)+p_{i}^{0}, \quad i=1,2, \ldots, k,
$$

and $r_{3}(t)$ is given by (51), $\tau_{3}(t)$ is given by (52). The behavior of a three-shock wave is illustrated in Fig. 12 . We see that shocks with higher amplitude move faster, at certain time all shocks merge to single one, shock profile becomes steeper with time and its amplitude eventually goes to zero.

(b) Generalized triangular wave solution of BE (36) is of the form (16) with $r(t)=r_{3}(t)$ and $\tau(t)=\tau_{3}(t), t>0$.

(c) Generalized $N$-wave solution of $\mathrm{BE}(36)$ for the over damping case can be written using formula (17).

(d) The forced $\mathrm{BE}(36)$ with initial conditions $U_{m}(x, 0)=-(m / x), m=1,2,3 \ldots$, has rational type solutions of the form

$$
U_{m}(x, t)=\frac{\dot{r}_{3}(t)}{r_{3}(t)} x-e^{-\gamma t}\left(\frac{r_{3}(0)}{r_{3}(t)}\right)\left(\frac{m H_{m-1}\left(\frac{r_{3}(0)}{r_{3}(t)} x, \frac{1}{2} \tau_{3}(t)\right)}{H_{m}\left(\frac{r_{3}(0)}{r_{3}(t)} x, \frac{1}{2} \tau_{3}(t)\right)}\right), \quad m=1,2,3, \ldots,
$$

where $r_{3}(t)$ and $\tau_{3}(t)$ are given by (51) and (52), respectively.

From the general form of the solution (53), clearly if the function $V(\eta(x, t), \tau(t))$, is bounded for $t \gg 1$, then the long-time behavior of $U(x, t)$ is described by $\lim _{t \rightarrow \infty} U(x, t)=\left(-\gamma / 2+\Omega^{\prime}\right) x$, and the limiting function is a stationary solution of the BE (36). 


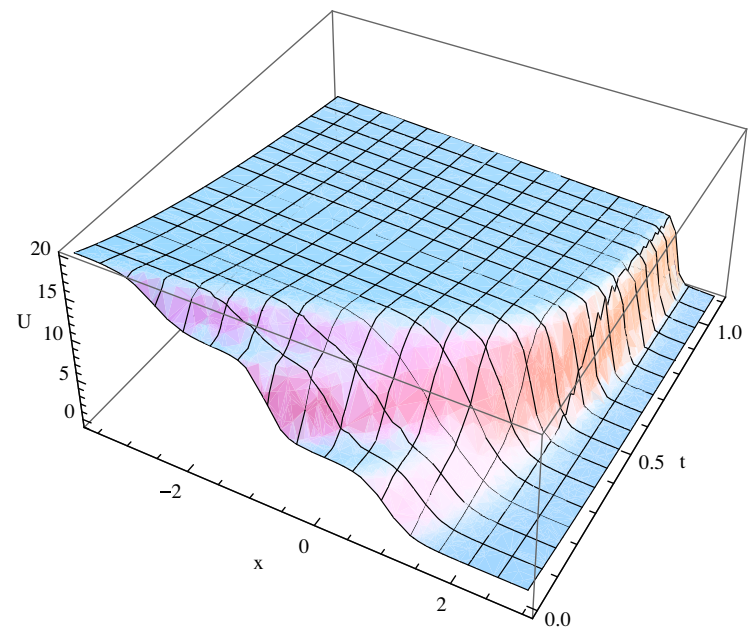

(a)

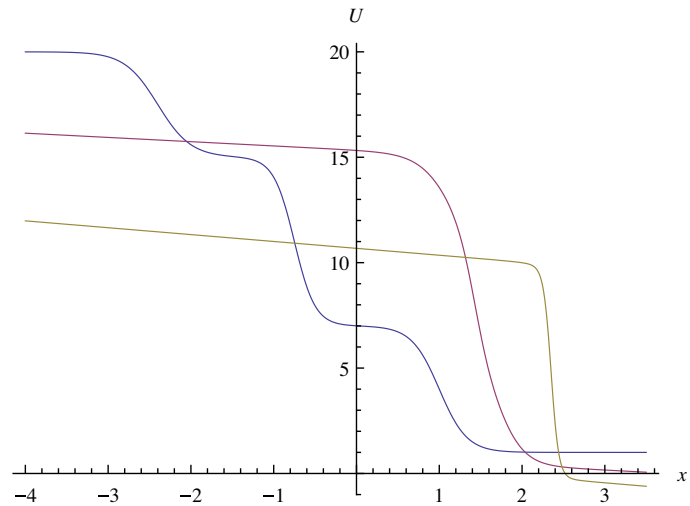

(b)

Fig. 12. Over damping case. (a) Fusion of a three-shock wave solution $U(x, t)$, when $\gamma=3, \omega_{0}=1, a_{1}=1, a_{2}=7, a_{3}=15, a_{4}=20$, $p_{1}^{0}=0, p_{2}^{0}=6, p_{3}^{0}=0, p_{4}^{0}=-12$. (b) Profiles of $U(x, t)$ at times $t=0, t=0.3, t=0.8$.

\subsubsection{Variable coefficient case with $\omega_{0}^{2}<0$}

In the study of damped harmonic oscillator, usually $\gamma$ and $\omega_{0}$ are positive parameters leading to the critical, under and the over damping cases, which we already discussed. However, we can take also $\omega_{0}^{2}=-\tilde{\omega}^{2}, \tilde{\omega}>0, \gamma>0$, and consider the Burgers equation

$$
U_{t}+\gamma U+U U_{x}=\frac{1}{2} e^{-\gamma t} U_{x x}+\tilde{\omega}^{2} x, \quad-\infty<x<\infty, t>0 .
$$

In that case, we have the IVP $\ddot{r}+\gamma \dot{r}-\tilde{\omega}^{2} r=0, r(0)=r_{0} \neq 0, \quad \dot{r}(0)=0$, which has solution

$$
r(t)=r_{0} \frac{\tilde{\omega}}{\tilde{\Omega}} e^{-\frac{\gamma t}{2}} \cosh [\tilde{\Omega} t+\tilde{\beta}],
$$

where $\tilde{\Omega}=\sqrt{\tilde{\omega}^{2}+\left(\gamma^{2} / 4\right)}$, and $\tilde{\beta}=\tanh ^{-1}(\gamma / 2 \tilde{\Omega})$. Then, BE (55) has solutions of the form,

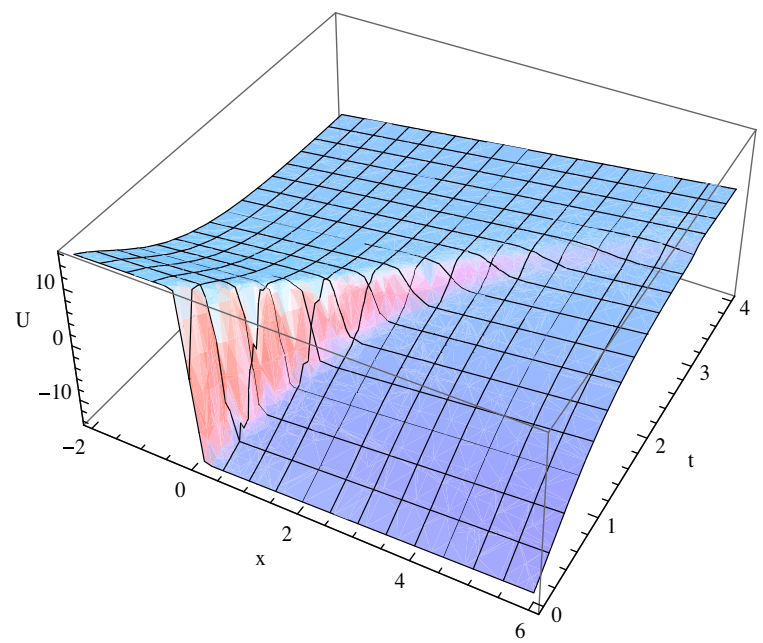

(a)

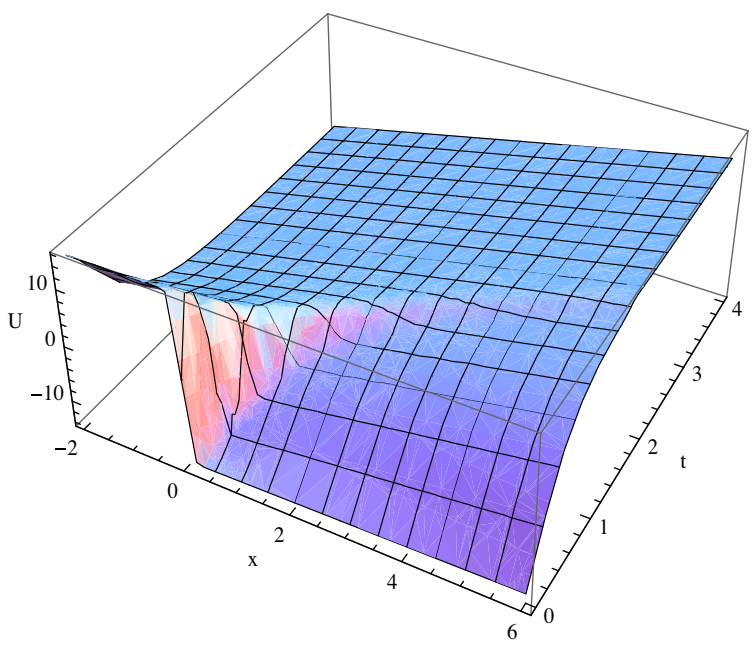

(b)

Fig. 13. (a) Shock wave solution when $\tilde{\omega}=1, \gamma=0.4, A=15, c=1$. (b) Shock wave solution when $\tilde{\omega}=2, \gamma=0.4, A=15, c=1$. 


$$
U(x, t)=(-(\gamma / 2)+\tilde{\Omega} \tanh (\tilde{\Omega} t+\tilde{\beta})) x+\frac{\tilde{\Omega}}{\tilde{\omega}}\left(\frac{e^{-\gamma t / 2}}{\cosh (\tilde{\Omega} t+\tilde{\beta})}\right) V\left(\frac{\tilde{\Omega}}{\tilde{\omega}}\left(\frac{e^{\gamma t / 2}}{\cosh (\tilde{\Omega} t+\tilde{\beta})}\right), \frac{\tilde{\Omega}}{\tilde{\omega}^{2}}\left(\tanh [\tilde{\Omega} t+\tilde{\beta}]-\frac{\gamma}{2 \tilde{\Omega}}\right)\right) .
$$

Notice that, when $\gamma \rightarrow 0$, one has $\tilde{\Omega} \rightarrow \tilde{\omega}$, and $\tilde{\beta} \rightarrow 0$. If $V(\eta, \tau)$ is as in the previous cases, then one can see that the solution (56) of the variable parametric $\mathrm{BE}$ (55) approaches the solution (31) of the constant coefficient forced $\mathrm{BE}$ (30), which in turn approaches the standard BE solution, when $\tilde{\omega} \rightarrow 0$.

Different type of exact solutions can be found and analyzed following the procedure in Section 2 . To illustrate the effect of increasing the parameter $\tilde{\omega}$, as an example, we plot shock wave solution for $\tilde{\omega}=1$, and for $\tilde{\omega}=2$. We observe that in Fig. 13b, the center of the wave profile moves faster and the shock contribution decays more rapidly to zero, comparing with the wave in Fig. 13a.

\section{Conclusion}

In this article, exactly solvable forced Burgers equations with specific time variable coefficients are discussed. In particular, Burgers equations with constant damping, exponentially decaying diffusion coefficient and a forcing term linear in the space variable were treated explicitly. For these models we found generalized shock wave solutions which speed, steepness, and shock amplitude are functions of time. Special properties such as fusion of shocks in traveling wave solutions, and motion of pole singularities of rational type solutions were observed. In addition, we shortly discussed the limiting case of the parametric equations, and the long-time behavior of their solutions. Similarly, our results can be used to study variable parametric forced Burgers models related with the Sturm-Liouville problems for the classical orthogonal polynomials [25].

We note also that, there are different approaches to study the variable parametric Burgers problems posed in this article. The one, which we used here, is transforming the inhomogeneous Burgers equation with variable coefficients to a standard Burgers equation, and then applying Cole-Hopf linearization. Another approach is a direct linearization of the variable parametric Burgers equation in the form of a variable parametric parabolic equation, which in turn can be transformed to a standard heat equation or can be solved using the evolution operator method. These problems are discussed in a forthcoming article.

As known, the Burgers equation has unique importance in computational physics due to the big number of applications to turbulence, shock formation, stochastic motion, etc. This is why we think that our solutions could have wide applications to real physical situations. Here we will mention only few potential fields. One interesting approach is related with quantum computational physics. A quantum algorithm as a microscopic-scale algorithm for a type-II quantum computer was studied by modeling the time evolution of a continuous field governed by the nonlinear Burgers equation in one space dimension [26]. In this case the Burgers equation is derived as an effective field theory governing the behavior of the quantum computer at its macroscopic scale, where both the lattice cell size and the time step interval become infinitesimal. If $U(2)$ quantum gate parameters are variable in space, then the Burgers equation with variable parameters, like tunable shear viscosity, will appear as effective description of quantum computer with control on quantum gates. It will require extension of techniques in [27] to quantum models with sufficiently many qubits per lattice node.

One of the most interesting applications of Burgers equation with external force is in stochastic processes. It was shown that the one-dimensional Burgers equation with an elastic forcing term is connected with the Ornstein-Uhlenbeck process [28]. The study of diffusion driven by the Burgers flow begins from first solving the Burgers equation for a chosen external force field and it is the place where our solutions could be explored. Next specifying the probability density, and ending with the corresponding "passive contaminant" concentration dynamics. Then solution of the Burgers equation with the forcing term, the Burgers velocity field, determines the stochastic Ito equation, where the given forced Burgers velocity field is perturbed by the noise term representing a molecular diffusion in the form of the Wiener process. The fluid velocity is considered as the forward drift of the stochastic process. For the time independent linear force term, the forward drift of the Markovian diffusion process is a linear function of $x$. It was proved that the forced Burgers dynamics and the diffusion-convection equation for the concentration of a passive component in a flow, in case of gradient velocity fields is compatible with the Markovian diffusion process input [29]. Solution of extended Burgers equation for time dependent force, considered in present paper, generalizes the usual one related with the Ornstein-Uhlenbeck process and depending on choice of the time depending function it can represent a stationary state or an anomalous spreading. This question is under investigation now.

Another interesting problem is the turbulence as was studied by Polyakov in terms of the Burgers equation, modeling the Navier-Stokes equation with white noise random force. It was shown that the model exhibits scaling behavior. If in our forced Burgers equation, $\omega^{2}(t)$ is a white noise force, then our solutions can be applied to analyze this problem. The advantage is that we can find an analytic solution for the arbitrary time dependent functions. This problem as well as the stretched structure of the solution, similar to stretched vortices in Navier-Stokes equation, is also under investigation.

As another application in stochastic mechanics, in [30] studying the limit of systems of Brownian particles with electrostatic repulsion when the number of particles increases to infinite, a non-linear stochastic differential equation has been introduced. Existence and uniqueness of the corresponding nonlinear diffusion process then has been related with real analyticity of solutions for the holomorphic Burgers equation. Generalization of the Burgers equation and its solution as given in the present paper could lead to interesting extension of these results. 
In plasma physics, an electrohydrodynamical model in the form of the Burgers equation with external force, describing the evolution of a local electric field in plasma under the assumption of motionless ions with electric field obeying the Poisson equation was proposed [31]. This is the electric analog of the magnetohydrodynamical problem considered by Olsen, where the external field in Burgers equation is determined by the magnetic field. In more general situation with allowed ions motion, this will lead to the Burgers equation with external force depending on space and time through electric and magnetic fields.

Last application which we mention is related with the study of unsaturated flow, in the presence of a web of plant roots, which was modeled by Burgers' equation with a spatially varying sink function [32].

We summarize and conclude by saying that our work was motivated by two main ideas. First one is the big number of possible applications in real physical problems, as mentioned above. And second one is the exact solvability of our models, where the explicitly found solutions can provide convenient schemes to develop perturbation theory, test numerical methods and develop new algorithms necessary for revealing the nature of many nonlinear physical phenomena.

\section{Acknowledgment}

This work is supported by TÜBITAK, Project No. 110 T679.

\section{References}

[1] Burgers JM. A mathematical model illustrating the theory of turbulence. Adv Appl Mech 1948;1:171

[2] Burgers JM. The nonlinear diffusion equation. Boston: Reidel; 1974.

[3] Bec J, Khanin K. Burgers turbulence. Phys Rep 2007;447:1.

[4] Cole JD. On a quasi-linear parabolic equation occurring in aerodynamics. Quart Appl Math 1951;9:225.

[5] Hopf E. The partial differential equation $u_{t}+u u_{x}=u_{x x}$. Comm Pure Appl Math 1950;3:201.

[6] Lighthill M]. In surveys in mechanics. Cambridge University Press; 1956.

[7] Woyczynski WA. Burgers-KPZ turbulence. Gttingen lectures. Springer; 1998.

[8] Guixu Z, Zhibin L, Yishi D. Exact solitary wave solutions of nonlinear wave equations. Sci China A 2001:44(3):396.

[9] Bai Cheng-Lin, Zhao Hong. Infinitely many new solutions and the closed form of the solution for initial-value problem of the Burgers equation. Chaos Solitons Fractals 2007;33:1285.

[10] Whitham GB. Linear and nonlinear waves. John Wiley and Sons Inc.; 1999.

[11] Wang S, Tang X, Lou SY. Soliton fission and fusion: Burgers equation and Sharma-Tasso-Olver equation. Chaos Solitons Fractals 2004;21:231.

[12] Choodnovsky DV, Choodnovsky GV. Pole expansions of nonlinear partial differential equations. Il Nuovo Cimento 1977;40(2):339.

[13] Calogero F. Motion of poles and zeros of special solutions of nonlinear and linear partial differential equations and related solvable many-body problems. Il Nuovo Cimento 1978;43(2):177.

[14] Deconinck B, Kimura Yo, Segur H. The pole dynamics of rational solutions of the viscous Burgers equation. J Phys A Math Theor 2007;40:5459.

[15] Orlowski A, Sobczyk K. Solitons and shock waves under random external noise. Rep Math Phys 1989;27:59.

[16] Moreau E, Vallee O. Connection between the Burgers equation with an elastic forcing term and a stochastic process. Phys Rev E 2006;73:016112.

[17] Eule S, Friedrich R. A note on the forced Burgers equation. Phys Lett A 2006;351:238.

[18] Schulze-Halberg A. New exact solutions of the non-homogeneous Burgers equation in $(1+1)$ dimensions. Phys Scripta 2007;75:531.

[19] Zola RS, Dias JC, Evangelista LR, Lenzi MK, Silva LR. Exact solutions for a forced Burgers equation with a linear external force. Physica A 2008;387:2690.

[20] Sophocleous C. Transformation properties of a variable-coefficient Burgers equation. Chaos Solitons Fractals 2004;20:1047.

[21] Xu T, Zhang C, Li J, Meng X, Zhu H, Tian Bo. Symbolic computation on generalized Hopf Cole transformation for a forced Burgers model with variable coefficients from fluid dynamics. Wave Motion 2007;44:262.

[22] Chidella SR, Yadav MK. Large time asymptotics for solutions to a nonhomogeneous Burgers equation. Appl Math Mech Engl Ed 2010;31:1198.

[23] Schulze-Halberg A, Jimenez JM. Darboux transformations for the time-dependent nonhomogeneous Burgers equation in $(1+1)$ dimensions. Phys Scripta 2009;80:065014.

[24] Dattoli G, Ottaviani PL, Torre A, Vazquez L. Evolution operator equations: integration with algebraic and finite-difference methods. Applications to physical problems in classical and quantum mechanics and quantum field theory. Rivista Del Nuovo Cimento 1997;20:2.

[25] Büyükaşık ŞA, Pashaev OK, Ulaş-Tigrak E. Exactly solvable quantum Sturm-Liouville problems. J Math Phys 2009;50:072102.

[26] Yepez J. Quantum lattice-gas model for the Burgers equation. J Stat Phys 2002;107:203.

[27] Yepez J. Open quantum system model of the one-dimensional Burgers equation with tunable shear viscosity. Phys Rev A 2006:042322.

[28] Garbaczewski P. Comment on connection between the Burgers equation with an elastic forcing term and a stochastic process. arXiv:cond-mat/ $0605505 \mathrm{v} 1$.

[29] Garbaczewski P, Kondrat G, Olkiewicz R. Burgers velocity field and the electromagnetic forcing in Schrödinger's interpolating dynamics. arXiv:chaodyn/9603018v1.

[30] Bonami A, Bouchut F, Cépa E, Lépingle D, Transform H. A non-linear stochastic differential equation involving the Hilbert transform. J Funct Anal 1999;165:390.

[31] Moreau E, Vallee O. The Burgers equation as electrohydrodynamic model in plasma physics. arXiv:physics/0501019.

[32] Broadbridge P. The forced Burgers equation, plant roots and Schrödinger's eigenfunctions. J Eng Math 1999;36:25. 